\title{
A General Closed Form Approximation Pricing Formula for Basket and Multi-Asset Spread Options*
}

\author{
Tommaso Pellegrino \\ Quantitative Strategist in the Strats Team, Mercuria UK LLP, London, UK \\ Email: tommaso.pellegrino.quant@gmail.com
}

How to cite this paper: Pellegrino, $\mathrm{T}$. (2016) A General Closed Form Approximation Pricing Formula for Basket and Multi-Asset Spread Options. Journal of Mathematical Finance, 6, 944-974. http://dx.doi.org/10.4236/jmf.2016.65063

Received: October 13, 2016

Accepted: November 26, 2016

Published: November 30, 2016

Copyright $\odot 2016$ by author and Scientific Research Publishing Inc. This work is licensed under the Creative Commons Attribution International License (CC BY 4.0).

http://creativecommons.org/licenses/by/4.0/

\section{(c) (i) Open Access}

\begin{abstract}
The aims of this paper are twofold. Firstly, we present an approximating formula for pricing basket and multi-asset spread options, which genuinely extends Caldana and Fusai's (2013) two-asset spread options formula. Secondly, under the lognormal setting, we show that our formula becomes a Black and Scholes type formula, extending Bjerksund and Stensland's (2011). Numerical experiments and comparison with Monte Carlo simulations and other methods available in the literature are discussed. The main contribution of this paper is to provide practitioners with a pricing formula, which can be used for pricing basket and multi-asset spread options, even under a non-Gaussian framework.
\end{abstract}

\section{Keywords}

Basket Options, Spread Options, Derivatives Pricing, Characteristic Function, Fourier Inversion

\section{Introduction}

Multi-asset spread options (or basket-spread options) are options whose payoff at maturity is given by the difference (or so-called the spread) between two baskets of aggregated asset prices. For a standard European multi-asset spread call option, the payoff function reads:

$$
\operatorname{Payoff}(\mathrm{w}, S, K, T, M, N)=\left(\sum_{k=1}^{M} w_{k} S_{k}(T)-\sum_{k=M+1}^{M+N} w_{k} S_{k}(T)-K\right)^{+},
$$

\footnotetext{
${ }^{\star}$ The views, opinions, positions, results or strategies expressed in this article are those of the author and do not represent the views, opinions, positions, results or strategies of, and should not be attributed to, Mercuria UK LLP.
} 
where $K$ is the strike price, $S_{k}(T)$ is the $k^{\text {th }}$ underlying asset price at maturity $t=T$ and where $\mathrm{w}=\left(w_{1}, \cdots, w_{N+M}\right)^{\mathrm{T}} \in \mathbb{R}_{+}^{N+M}$ is a vector of weights.

This embodies a general class of options, including the two-asset spread $(M=N=1)$, basket $(N=0)$ and single-asset vanilla $(M=1, N=0)$ options.

Multi-asset spread options are prevalent in a variety of markets, including the fixed income, foreign exchange, commodity, energy and equity markets. They are useful financial tools for hedging a portfolio of long and short positions in the underlying assets. A simple, accurate and efficient method to price and hedge multi-asset spread options is therefore inevitable.

In most contributions from the literature on basket and multi-asset spread option pricing, the underlying asset prices are assumed to follow lognormal processes. However, the celebrated Black and Scholes (1973) formula in [1] cannot be easily extended to the basket and multi-asset spread options case since the linear combination of lognormal random variables does not follow the lognormal distribution. The lack of an exact marginal distribution for the multi-asset spread (i.e. $\left.\sum_{k=1}^{M} w_{k} S_{k}(T)-\sum_{k=M+1}^{M+N} w_{k} S_{k}(T)\right)$ refrains the emergence of an exact closed form solution for the multi-asset spread option. Several approaches have been proposed to tackle the problem, including Monte Carlo simulations, tree-based methods, partial differential equations and analytical approximations. The last category is the most appealing one, as the other methods, although more robust, are less efficient and computationally expensive due to the large dimension of the pricing problem.

If we consider first the case of two-asset spread options, namely $M=N=1$ in Equation (1), under the Black and Scholes framework, Carmona and Durrleman in [2] have derived a semi-analytic formula to approximate the two-asset spread option price and later, the same authors have extended their analysis to deal with multi-asset spread options, see [3]. However, before their semi-analytic formula can be employed, a set of couple non-linear equations needs to be solved numerically. As pointed out by Deng et al. in [4], the convergence of such numerical solution was very sensitive to the choice of initial input values. The most notable analytic solution appears to be given by Kirk in [5], in which an analytic formula is proposed to approximate the two-asset spread option price. It remains as one of the most popular methods among practitioners, because it retains all the simplicity and tractability of the classical Black and Scholes formula. Kirk's formula has been extended (but only for the particular case where $M=1$ and $N=$ 2) by Alòs et al. in [6] and by Lau and Lo in [7] in the more general cases where $M>1$ and $N>1$. Another notable analytic solution has been recently proposed by Deng et al. in [8], in which they introduced the notion of exercise boundary and approximated it with a quadratic function (instead of using a linear function as in [5]), such that the option price could still be valued analytically. The same authors, see [4], have extended their analysis to the pricing of multi-asset spread options, however, they only provided a solution for the special case $M=1$. For $M>1$, they suggested approximating the long basket variable, i.e. $\sum_{k=1}^{M} w_{k} S_{k}(T)$ with its geometric average. As pointed out in [7], such crude approximation does not only deteriorate the pricing accuracy but also 
refrains their solution to be applicable for the pricing of basket options (i.e. when $M>1, N=0$ ).

If we consider then the case of multi-asset spread options, in a lognormal setting, besides to what it has been already said above, we can mention the work of Borovkova et al. in [9], where the authors propose to approximate the multi-asset spread option distribution with a shifted lognormal distribution by matching their first three moments. But again, the matching process requires numerically solving a set of non-linear equations. Two additional closed form approximations' formulas for multi-asset spread options have been recently proposed by Pellegrino and Sabino in [10] and in [11]. However, the authors focused only on the particular case where $M=1$ and $N=2$.

For the basket option pricing case (where $M>1$ and $N=0$ in Equation (1)), under the assumptions that the dynamics of the underlying prices follows a multivariate geometric Brownian motion, several accurate analytical approximations are available, among which we can mention the works of Curran in [12], Beisser in [13], who extended to basket options the original idea of Rogers and Shi (see [14]) for Asian options, Gentle in [15], who extended to basket options the original idea of Vorst (see [16]) for Asian options (replacement of the arithmetic average by a geometric average and strike adjustment for the difference between the two quantities), Levy in [17], where the distribution of the basket is approximated by a lognormal distribution, such that its first two moments coincide with those of the original distribution, Ju in [18], who considered a Taylor expansion of the ratio of the characteristic function of the arithmetic average to that of the approximating lognormal random variable around zero volatility, Milevsky and Posner in [19], who used the reciprocal Gamma distribution as an approximation for the distribution of the basket and in [20], who used distributions from the Johnson's family (see [21]) as state-space densities to match the higher moments of the arithmetic mean distribution. Analytical approximations based on the concept of co-monotonicity are also available in the literature, see for example [22] [23] and [24].

Few results are available in the literature concerning the pricing of multi-asset spread options under a non-Gaussian setting, as pointed out in [25] and in [26]. A Fourier transform was originally proposed by Dempster and Hong in [27], who implement a valuation method based on the Fast Fourier Transform (FFT), applying the original idea of Carr and Madan, see [28]. An FFT technique is also applied by Hurd and Zhou in [29], who propose a pricing method based on an explicit formula for the Fourier transform of the spread option payoff in term of the gamma function. Their formula requires a bivariate Fourier inversion.

A noticeable work on the pricing of two-asset spread options under the non-Gaussian framework is the one of Caldana and Fusai in [25], where the authors derive the Bjerksund and Stensland formula (see [30]), but for general processes. Indeed, the only quantity which has to be known explicitly is the joint characteristic function of the log-returns of the two assets. The computation of their lower bound requires only a univariate Fourier inversion, as opposed to the bivariate inversion required in [27] and [29], implying that the computation is much faster. 
Caldana et al. in [26] have recently tackled the problem to extend the approach in [25] to deal with basket and multi-asset spread options under a very general dynamics for the underlying prices. In particular, the authors propose two kinds of approximations: an accurate lower bound based on an approximating set, which involves a univariate Fourier inversion and an optimization with respect a particular parameter and a fast bounded approximation based on the arithmetic-geometric average inequality, which generalizes the approach in [16]. In particular, for the geometric Brownian motion case, the second approximating formula in [26] coincides with the one in [15].

The aims of this paper are twofold. Firstly, we present a general closed form approximation formula for the pricing of multi-asset spread options, which genuinely extends the one in [25] for two-asset spread options. Indeed our approach does not require any optimization step (in contrast with the first approximation formula in [26]) and it is based only on a univariate Fourier inversion. Furthermore, the approach presented in this paper goes beyond the classical Black and Scholes framework, since it can be applied to models for which the joint characteristic function of the log-returns for the underlying assets is known analytically. Secondly, under the lognormal setting, we show that the general approximation formula becomes a Black and Scholes type formula, extending the Bjerksund and Stensland pricing formula in [30] to the multi-asset spread options pricing problem in the same way as Lau and Lo in [7] extended Kirk's formula. Numerical experiments are discussed and a comparison with Monte Carlo simulations and with the other methods available in the literature is performed.

The main contribution of this paper is to provide practitioners with a general closed form approximation pricing formula, which can be used for real-time pricing of multi-asset spread options, even under a non-Gaussian framework.

The rest of the paper is outlined as follows: in Section 2 we present the general closed form approximation pricing formula for multi-asset spread options. This is done via a procedure, which requires only a univariate Fourier inversion and it is applicable to models for which the joint characteristic function of the underlying assets is known in closed form. This approach has been proposed by Caldana and Fusai in [25] when pricing two-asset spread options. In Section 3, we show that if we assume a lognormal dynamics for the underlying asset prices, then the general closed form approximation formula presented in Section 2 becomes a Black and Scholes type formula, which extends the one in [30] for two-asset spread options, since the way it is derived uses the same insight as the one originally proposed by Bjerksund and Stensland in [30]. In Section 4 we present two non-Gaussian models on which the results exposed in Section 2 are applied. Numerical experiments for the geometric Brownian motion case and for the non-Gaussian models are discussed in Section 5 for both basket and multi-asset spread options. Section 6 concludes the paper.

\section{A General Closed Form Approximation Pricing Formula for Basket and Multi-Asset Spread Options}

In this section we present the general closed form approximation formula for the pric- 
ing of basket and multi-asset spread options. The approach extends the one in [25] for options written on the spread between two assets.

In particular, define the event $A$ as follows:

$$
A \equiv\left\{\omega: \frac{\mathrm{e}^{\tilde{F}} \prod_{k=1}^{M} S_{k}(T, \omega)^{b_{k}}}{\mathbb{E}^{\mathbb{Q}}\left[\prod_{k=1}^{M} S_{k}(T, \omega)^{b_{k}}\right]}>\frac{\mathrm{e}^{\tilde{K}} \prod_{k=M+1}^{M+N} S_{k}(T, \omega)^{b_{k}}}{\mathbb{E}^{\mathbb{Q}}\left[\prod_{k=M+1}^{M+N} S_{k}(T, \omega)^{b_{k}}\right]}\right\},
$$

where

$$
\begin{aligned}
& \tilde{F} \equiv \ln \left(\sum_{k=1}^{M} w_{k} F_{k}(t, T, \omega)\right) \\
& \text { and } \\
& \tilde{K} \equiv \ln \left(\sum_{k=M+1}^{M+N} w_{k} F_{k}(t, T, \omega)+K\right),
\end{aligned}
$$

and where the coefficients $b_{1}, \cdots, b_{M}, b_{M+1}, \cdots, b_{M+N}$ are defined as follows:

$$
\left\{\begin{array}{l}
b_{k} \equiv \frac{w_{k} F_{k}(t, \tilde{T})}{\mathrm{e}^{\tilde{F}}}, \text { for } k=1, \cdots, M, \\
b_{k} \equiv \frac{w_{k} F_{k}(t, \tilde{T})}{\mathrm{e}^{\tilde{K}}}, \text { for } k=M+1, \cdots, M+N .
\end{array}\right.
$$

In what follows, for sake of simplicity in the notation, we will drop the explicit dependency on $\omega$ for the spot prices $S_{k}(t)$, for $k=1, \cdots, M+N$.

Let $X_{k}(T)$ be the log-return over the period $[t, T]$ :

$$
X_{k}(T)=\ln \left(\frac{S_{k}(T)}{S_{k}(t)}\right) \text {, for } k=1, \cdots, M+N .
$$

We assume that the joint characteristic function of the $M+N$ stock returns, under the risk-neutral measure $\mathbb{Q}$, is known:

$$
\phi_{T}=\mathbb{E}^{\mathbb{Q}}\left[\exp \left(i \sum_{k=1}^{M+N} u_{k} X_{k}(T)\right)\right], \quad \text { where } \mathrm{u}=\left(u_{1}, u_{2}, \cdots, u_{N+M}\right)^{\mathrm{T}} \in \mathbb{R}^{M+N} .
$$

The main result is stated in the following proposition, for which a proof is reported in Appendix A.1.

Proposition 1 (Closed Form Approximation Pricing Formula for Multi-Asset Spread Options) The price $C_{K}(t, T)$ at time $t$ of a multi-asset spread call option, whose payoff is given by (1), with strike price $K$ and maturity $T$ can be approximated by $C_{K}(t, T)^{E C F}$, which is defined as follows.

$$
\begin{aligned}
C_{K}(t, T)^{E C F} & =C_{K}^{E C F}(t, T, \tilde{F}, \tilde{K}, \boldsymbol{b}) \\
& =\frac{\mathrm{e}^{-r(T-t)-\alpha k}}{\pi} \int_{0}^{+\infty} \mathrm{e}^{-i \gamma k} \Psi_{T}(\gamma ; \alpha, \tilde{F}, \tilde{K}, \boldsymbol{b}) \mathrm{d} \gamma,
\end{aligned}
$$

where for an opportune damping coefficient $\alpha>0$, the function $\Psi_{T}(\gamma ; \alpha, \tilde{F}, \tilde{K}, \boldsymbol{b})$ is defined as follows: 


$$
\begin{aligned}
& \Psi_{T}(\gamma ; \alpha, \tilde{F}, \tilde{K}, \boldsymbol{b})=\frac{\exp \left(i(\gamma-\alpha i)\left(\tilde{F}+\ln \left(\frac{\phi_{T}\left(0, \cdots, 0,-i b_{M+1}, \cdots,-i b_{M+N}\right)}{\phi_{T}\left(-i b_{1}, \cdots,-i b_{M}, 0, \cdots, 0\right)}\right)\right)\right)}{i(\gamma-\alpha i)} \\
& \times\left(\sum_{j=1}^{M} w_{j} \phi_{T}\left((\gamma-\alpha i) b_{1}-i \delta_{1 j}, \cdots,(\gamma-\alpha i) b_{M}-i \delta_{M j},-(\gamma-\alpha i) b_{M+1}, \cdots,-(\gamma-\alpha i) b_{M+N}\right)\right. \\
& -\sum_{j=M+1}^{M+N} w_{j} \phi_{T}\left((\gamma-\alpha i) b_{1}, \cdots,(\gamma-\alpha i) b_{M},-(\gamma-\alpha i) b_{M+1}-i \delta_{(M+1) j}, \cdots,-(\gamma-\alpha i) b_{M+N}-i \delta_{(M+N) j}\right) \\
& \left.-K \phi_{T}\left((\gamma-\alpha i) b_{1}, \cdots,(\gamma-\alpha i) b_{M},-(\gamma-\alpha i) b_{M+1}, \cdots,-(\gamma-\alpha i) b_{M+N}\right)\right)
\end{aligned}
$$
where $\delta_{i j}$ is the Kronecker delta, where $\tilde{F}, \tilde{K}$ as well as the coefficients $\boldsymbol{b}=\left(b_{1}, \cdots, b_{M+N}\right)^{\mathrm{T}}$ are defined in Equations (3), (4) respectively and where ECF stands for Extended Caldana and Fusai.

Proof. See Appendix A.1.

Some comments on the above approximation formula are due.

First, if we look at Equation (6), in order to compute the price of the multi-asset spread call option, a univariate Fourier inversion is required. The damping coefficient $\alpha>0$ has to be introduced to ensure the existence of the Fourier transform in line with the original remark in [28], as the call pricing function is not square-integrable. The integral in Equation (6) can be computed using standard numerical quadratures or via an FFT algorithm.

Second, if the characteristic function $\phi_{T}$ is known in closed form, then the Fourier transform of the modified multi-asset spread call option price can be expressed in terms of the complex function $\Psi_{T}$. In particular, we do not require the characteristic function to be exponential affine with respect to the initial value of the state variables.

Moreover, the a priori choice for $\tilde{F}, \tilde{K}$ as well as for the coefficients $b_{1}, \cdots, b_{M+N}$ in Equations (3), (4) generalizes the one done for two-asset spread options in [30] under the Black and Scholes framework and in [25] for the non-Gaussian case and avoids solving an optimization problem in order to compute the price. Indeed, in theory, one could maximize the value of the option with respect to these parameters. However, as pointed out in [25], this is not necessary because the above choice turn out to be very efficient for most part of practical problems, as it will be shown in the numerical experiments in Section 5. Besides that, performing an optimization with respect to the unknown parameters $\tilde{F}, \tilde{K}$ and $\boldsymbol{b}$ could slow down very significantly the computational speed of the proposed method, as the number of parameters to be optimized increases linearly with the dimension of the pricing problem.

The approximation can also be applied to the Greeks computation. In particular, assuming that interchange of differentiation and integration is allowed, the formula for the first-order sensitivity of the multi-asset spread option price to a change in the spot price of a generic asset is given by

$$
\frac{\partial C_{K}(t, T, \tilde{F}, \tilde{K}, \mathrm{~b})^{E C F}}{\partial S_{k}}=\frac{\mathrm{e}^{-r(T-t)-\alpha k}}{\pi} \int_{0}^{+\infty} \mathrm{e}^{-i \gamma k} \frac{\partial \Psi_{T}(\gamma ; \alpha, \tilde{F}, \tilde{K}, \mathrm{~b})}{\partial S_{k}} \mathrm{~d} \gamma,
$$


Similar formulas can be computed for the other Greeks but, as pointed out in [26], there is no guarantee that the above derivative will provide a lower bound to the true Delta.

We conclude this section by showing how the above approximation formula can be adapted for the pricing of basket $(M>0, N \equiv 0)$ and two-asset $\operatorname{spread}(M=1, N=1)$ options.

In particular, if we assume $M>0, N \equiv 0$, then the payoff function of Equation (1) reads as:

$$
\operatorname{Payoff}(\mathrm{w}, \mathrm{S}, K, T, M, 0)=\left(\sum_{k=1}^{M} w_{k} S_{k}(T)-K\right)^{+} \text {, }
$$

which is the well-known payoff function for a basket call option.

Then the following corollary holds.

Corollary 1 (Closed Form Approximation Pricing Formula for Basket Options). The price $C_{K}(t, T)$ at time $t$ of a basket call option, whose payoff is given by (9), with strike price $K$ and maturity $T$ can be approximated by $C_{K}^{E C F}(t, T, \tilde{F}, \tilde{K}, \boldsymbol{b})$, which is defined as follows:

$$
C_{K}^{E C F}(t, T, \tilde{F}, \tilde{K}, \boldsymbol{b})=\frac{\mathrm{e}^{-r(T-t)-\alpha k}}{\pi} \int_{0}^{+\infty} \mathrm{e}^{-i \gamma k} \Psi_{T}(\gamma ; \alpha, \tilde{F}, \tilde{K}, \boldsymbol{b}) \mathrm{d} \gamma,
$$

where for an opportune damping coefficient $\alpha>0$, the function $\Psi_{T}(\gamma ; \alpha, \tilde{F}, \tilde{K}, \boldsymbol{b})$ is defined as follows:

$$
\begin{aligned}
& \Psi_{T}(\gamma ; \alpha, \tilde{F}, \tilde{K}, \boldsymbol{b})=\frac{\exp \left(i(\gamma-\alpha i)\left(\tilde{F}-\ln \left(\phi_{T}\left(-i b_{1}, \cdots,-i b_{M}\right)\right)\right)\right)}{i(\gamma-\alpha i)} \\
& \times\left(\sum_{j=1}^{M} w_{j} \phi_{T}\left((\gamma-\alpha i) b_{1}-i \delta_{1 j}, \cdots,(\gamma-\alpha i) b_{M}-i \delta_{M j}\right)-K \phi_{T}\left((\gamma-\alpha i) b_{1}, \cdots,(\gamma-\alpha i) b_{M}\right)\right)
\end{aligned}
$$

where $\delta_{i j}$ is the Kronecker delta and where $\tilde{F}, \tilde{K}$ as well as the coefficients $\boldsymbol{b}=\left(b_{1}, \cdots, b_{M}\right)^{\mathrm{T}}$ are defined in Equations (3), (4) respectively.

Proof. The result follows by repeating the proof in Proposition 1 assuming $N \equiv 0$.

If we assume $M=1, N=1$, then the payoff function of Equation (1) reads as:

$$
\text { Payoff }(\mathrm{w}, \mathrm{S}, K, T, 1,1)=\left(w_{1} S_{1}(T)-w_{2} S_{2}(T)-K\right)^{+} \text {, }
$$

which is the well-known payoff function for a two-asset spread call option.

The following corollary shows that in this particular case our formula coincides with the one in [25].

Corollary 2 (Closed Form Approximation Pricing Formula for Two-Asset Spread Options). The price $C_{K}(t, T)$ at time $t$ of a two-asset spread call option, whose payoff is given by (12), with strike price $K$ and maturity $T$ can be approximated by $C_{K}^{C F}(t, T, \tilde{F}, \tilde{K}, b)$, which is defined as follows:

$$
C_{K}^{C F}(t, T, \tilde{F}, \tilde{K}, \boldsymbol{b})=\frac{\mathrm{e}^{-r(T-t)-\alpha k}}{\pi} \int_{0}^{+\infty} \mathrm{e}^{-i \gamma k} \Psi_{T}(\gamma ; \alpha, \tilde{F}, \tilde{K}, \boldsymbol{b}) \mathrm{d} \gamma,
$$


where for an opportune damping coefficient $\alpha>0$, the function $\Psi_{T}(\gamma ; \alpha, \tilde{F}, \tilde{K}, \tilde{\boldsymbol{b}})$ is defined as follows:

$$
\begin{aligned}
\Psi_{T}(\gamma ; \alpha, \tilde{F}, \tilde{K}, \boldsymbol{b})= & \frac{\exp \left(i(\gamma-\alpha i)\left(\ln \phi_{T}\left(0,-i b_{2}\right)\right)\right)}{i(\gamma-\alpha i)}\left(w_{1} \phi_{T}\left((\gamma-\alpha i)-i,-(\gamma-\alpha i) b_{2}\right)\right. \\
& \left.-w_{2} \phi_{T}\left((\gamma-\alpha i),-(\gamma-\alpha i) b_{2}-i\right)-K \phi_{T}\left((\gamma-\alpha i),-(\gamma-\alpha i) b_{2}\right)\right)
\end{aligned}
$$

where $\tilde{F} \equiv \ln \left(w_{1} F_{1}(t, T)\right), \tilde{K}=\ln \left(w_{2} F_{2}(t, T)+K\right)$ and the coefficients $\boldsymbol{b}=\left(b_{1}, b_{2}\right)^{\mathrm{T}}$ are respectively $b_{1} \equiv 1$ and $b_{2}=w_{2} F_{2}(t, T) / \mathrm{e}^{\tilde{K}}$ and where CF stands for Caldana and Fusai.

Proof. The result follows by repeating the proof in Proposition 1 assuming $M \equiv 1$ and $N \equiv 1$.

As mentioned in Section 1, a first attempt to extend the approach in [25] to deal with multi-asset spread (and basket) options is reported in [26].

In particular, the starting point of the authors is to consider the geometric average of the underlying prices

$$
G_{N+M}(T) \equiv \prod_{k=1}^{N+M} S_{k}(T)^{w_{k}}
$$

where no assumption on the sign of the $w_{k}$, for $k=1, \cdots, N+M$, is made.

Then, they define a feasible but sub-optimal exercise strategy by looking at the set $\mathcal{G}:$

$$
\mathcal{G}=\left\{\omega: \ln G_{N+M}(T)>\chi\right\}=\left\{\omega: Y_{N+M}(T)>\chi\right\},
$$

for an opportune parameter $\chi$ which will be defined later.

The lower bound $C_{K}^{\mathcal{G}}(t)$ in [26] for the multi-asset spread option pricing is therefore defined by:

$$
C_{K}^{\mathcal{G}}(t)=\mathrm{e}^{-r(T-t)} \mathbb{E}^{\mathbb{Q}}\left[\text { Payoff }(\mathrm{w}, \mathrm{S}, K, T, M, N) \mathbb{1}_{\mathcal{G}}\right]
$$

and the explicit computation is given in the following proposition, see [26], Proposition 1.

Proposition 2 (Caldana et al. (2016) Lower Bound for Multi-Asset Spread Options). Let $\alpha>0$ denote an opportune damping coefficient and assume that

$$
\left\{e_{k}, \alpha w+e_{k}\right\} \subset \mathcal{A}_{X}, \forall k=1, \cdots, M+N,
$$

where $e_{k}$ denotes the $k^{\text {th }}$ element of the canonical basis in $\mathbb{R}^{N+M}$ and where $\mathcal{A}_{\boldsymbol{X}}$ is the interior of the set

$$
\left\{v \in \mathbb{R}^{N+M}: \mathbb{E}^{\mathbb{Q}}\left[\exp \left(i \sum_{k=1}^{N+M} v_{k} X_{k}(T)\right)<+\infty\right]\right\}
$$

Then, the price $C_{K}(t, T)$ at time t of a multi-asset spread call option, whose payoff is given in Equation (1), with strike price $K$ and maturity $T$ can be approximated by $C_{K}^{\mathcal{G}}(t)$, which is defined as follows:

$$
C_{K}^{\mathcal{G}}(t)=\max _{\chi \in \mathbb{R}} C_{K}^{\mathcal{G}}(t, \chi),
$$


where

$$
C_{K}^{\mathcal{G}}(t, \chi)=\frac{\mathrm{e}^{-\alpha \chi-r(T-t)}}{\pi} \int_{0}^{+\infty} \mathrm{e}^{-i \gamma \chi} \Psi_{T}(\gamma ; \alpha) \mathrm{d} \gamma
$$

where

$$
\begin{aligned}
& \Psi_{T}(\gamma ; \alpha) \\
& =\frac{1}{i \gamma+\alpha}\left[\sum_{k=1}^{M+N} w_{k} S_{k}(t) \Phi_{T}\left(\gamma-i \alpha,-i \boldsymbol{e}_{k}, \boldsymbol{w}, Y_{M+N}(t)\right)-K \Phi_{T}\left(\gamma-i \alpha, \mathbf{0}, \boldsymbol{w}, Y_{N+M}(t)\right)\right]
\end{aligned}
$$

and where the function $\Phi_{T}$ is the joint characteristic function of the log-returns and the log-geometric average:

$$
\begin{aligned}
\Phi_{T}\left(u_{0}, \boldsymbol{u}, \boldsymbol{w}, Y_{N+M}(t)\right) & \equiv \mathbb{E}^{\mathbb{Q}}\left[\exp \left(i \sum_{k=1}^{N+M} u_{k} X_{k}(T)+u_{0} Y_{N+M}(t)\right)\right] \\
& =\exp \left(i u_{0} Y_{N+M}(t)\right) \phi_{T}\left(\boldsymbol{u}+u_{0} \boldsymbol{w}\right) .
\end{aligned}
$$

Proof. See [26], Appendix 1.

As we can see from Equation (18), the computation of the lower bound in [26] requires a univariate Fourier inversion and an optimization with respect to the parameter $\chi$. This represents the main difference between our approach and the one in [26] as no optimization has to be performed to compute the option price in Equation (6). Besides that, our formula genuinely extends the one in [25] for two-asset spread. This is not the case for the lower bound in [26].

The second approximation formula discussed in [26] exploits the so-called arithmetic-geometric average inequality and consists in a generalization of the Vorst (1992)'s approach, see [16], to a characteristic function framework. It does not require any optimization step in contrast with the above lower bound. However, numerical experiments reported in [26], Section 5, show that the approximating formula based on the arithmetic-geometric average inequality is in general less accurate than the lower bound above.

\section{The Geometric Brownian Motion Case: The Extended Bjerksund and Stensland Pricing Formula}

This section discusses in more detail the geometric Brownian case. In particular, in what follows we will consider a multi-variate Black and Scholes model. The evolution of the underlying prices, under the risk-neutral measure $\mathbb{Q}$, is given by:

$$
\mathrm{dS}(t)=\operatorname{Diag}(\mathrm{S}(t))((r 1-\mathrm{q}) \mathrm{d} t+\sqrt{\Sigma} \mathrm{dW}(t)),
$$

where $r$ is the risk-free rate, $q$ is the vector of dividend yields for each asset, 1 is a vector whose entries are all equal to one, $\Sigma$ is the covariance matrix, and $W$ is an $(N+M)$ dimensional Brownian motion.

The risk-neutral joint characteristic function of the $N+M$ stock returns in the geometric Brownian motion case is given by: 


$$
\phi_{T}(\mathrm{u})=\exp \left(i \mathrm{u}^{\mathrm{T}} \mathrm{g}(T-t)-\frac{1}{2} \mathrm{u}^{\mathrm{T}} \Sigma \mathrm{u}(T-t)\right)
$$

where

$$
\mathrm{g}=r 1-\mathrm{q}-\frac{1}{2} \operatorname{Diag}(\Sigma)
$$

Expression (23) can be used to compute the closed form approximation formula presented in Section 2.

However, under the Black and Scholes framework, all formulas can be explicitly computed. In particular, in what follows we will derive the so-called Extended Bjerksund and Stensland pricing formula for multi-asset spread options, via the conditional expectation method. We are aware of a different derivation of this pricing formula, only valid for the particular case $M=1$ and $N=2$, based on the original idea of Bjerksund and Stensland in [30]. More details can be found in [31].

Before doing it, we give a bit of insight about the origins of this formula. If we consider the pricing of a two-asset spread option, then it can be proved that the Kirk's formula in [5] follows from the expectation

$$
C(t, T, K)^{\text {Kirk }}=\mathrm{e}^{-r(T-t)} \mathbb{E}^{\mathbb{Q}}\left[\left(S_{1}(T)-\frac{\mathrm{e}^{\tilde{K}} S_{2}(T)^{b_{2}}}{\mathbb{E}^{\mathbb{Q}}\left[S_{2}(T)^{b_{2}}\right]}\right)^{+} \mid \mathcal{F}_{t}\right],
$$

where $\tilde{K}=\ln \left(F_{2}(t, T)+K\right)$ and $b_{2}=F_{2}(t, T) / \mathrm{e}^{\tilde{K}}$.

Bjerksund and Stensland in [30] use this insight to obtain an alternative spread option approximation formula. In particular, they argue that the implicit exercise strategy given by the Kirk's formula is to exercise if and only if $S_{1}(T)$ exceeds a power function of $S_{2}(T)$. The authors utilize this feasible but non-optimal exercise strategy and express the future payoff of the two-asset spread option as

$$
\left(S_{1}(T)-S_{2}(T)-K\right) \times \mathbb{1}\left(S_{1}(T)-\frac{\mathrm{e}^{\tilde{K}} S_{2}(T)^{b_{2}}}{\mathbb{E}\left[S_{2}(T)^{b_{2}}\right]}\right),
$$

If we consider now the multi-asset spread options pricing problem, Kirk's formula has been extended to deal with more than two underlyings by Lau and Lo in [7] ${ }^{1}$.

However, the same reasoning as the one above can be applied. Indeed, it can be verified that the Extended Kirk pricing formula proposed by Lau and Lo in [7] follows from solving the following expectation

$$
C(t, T, K)^{\text {Extended Kirk }}=\mathrm{e}^{-r(T-t)} \mathbb{E}^{\mathbb{Q}}\left[\left(\frac{\mathrm{e}^{\tilde{F}} \prod_{k=1}^{M} S_{k}(T)^{b_{k}}}{\mathbb{E}^{\mathbb{Q}}\left[\prod_{k=1}^{M} S_{k}(T)^{b_{k}}\right]}-\frac{\mathrm{e}^{\tilde{K}} \prod_{k=M+1}^{M+N} S_{k}(T)^{b_{k}}}{\mathbb{E}^{\mathbb{Q}}\left[\prod_{k=M+1}^{M+N} S_{k}(T)^{b_{k}}\right]}\right] \mathcal{F}_{t}^{+}\right],
$$

where

\footnotetext{
${ }^{1}$ Alòs et al. in [6] have extended the Kirk's formula only for the particular case $M=1$ and $N=2$.
} 


$$
\tilde{F}=\ln \left(\sum_{k=1}^{M} F_{k}(t, T)\right), \tilde{K}=\ln \left(\sum_{k=M+1}^{M+N} F_{k}(t, T)+K\right),
$$

and where the coefficients $b_{1}, \cdots, b_{M}, b_{M+1}, \cdots, b_{M+N}$ are defined as follows:

$$
b_{k}=\frac{F_{k}(t, T)}{\mathrm{e}^{\tilde{F}}} \text {, for } k=1, \cdots, M \text {, and } b_{k}=\frac{F_{k}(t, T)}{\mathrm{e}^{\tilde{K}}} \text {, for } k=M+1, \cdots, M+N .
$$

Therefore, the idea behind the Extended Bjerksund and Stensland pricing formula is to use this feasible but non-optimal exercise strategy in order to price the multi-asset spread option. The final result is reported in the following proposition.

Proposition 3 (Bjerksund and Stensland Pricing Formula for Multi-Asset Spread Options). The price $C_{K}(t, T)$ at time $t$ of a multi-asset spread call option, whose payoff is given by (1), with strike price $K$ and maturity $T$ can be approximated by $C_{K}^{E B S}(t, T)$, which is defined as follows.

$$
\begin{aligned}
C_{K}^{E B S}(t, T)= & \mathrm{e}^{-r(T-t)}\left(\sum_{k=1}^{M} w_{k} S_{k}(t) \mathrm{e}^{\left(r-q_{k}\right)(T-t)} N\left(\sigma_{k} c_{k} \sqrt{T-t}-d\right)\right. \\
& \left.-\sum_{k=M+1}^{M+N} w_{k} S_{k}(t) \mathrm{e}^{\left(r-q_{k}\right)(T-t)} N\left(\sigma_{k} c_{k} \sqrt{T-t}-d\right)-K N(-d)\right)
\end{aligned}
$$

where $N(\cdot)$ denotes the cumulative distribution function for a standard normal variable, where the coefficients $c_{1}, \cdots, c_{N+M}$ and $d$ are defined as follows:

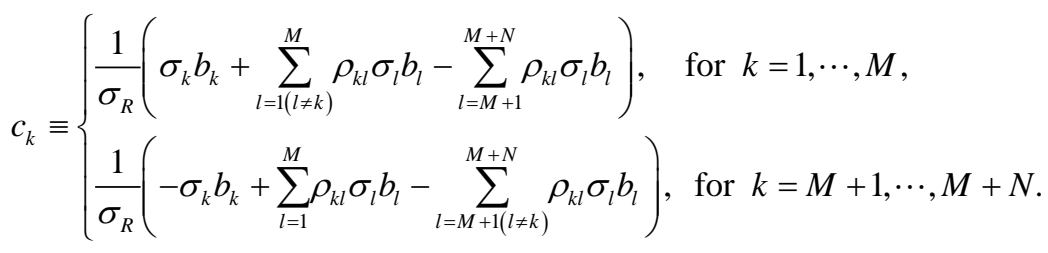

$$
\begin{aligned}
& d=\frac{1}{\sqrt{T-t} \sigma_{R}}\left(\tilde{K}-\tilde{F}-\ln \left(R(t) \mathbb{E}^{\mathbb{Q}}\left[\prod_{k=M+1}^{M+N} S_{k}(T)^{b_{k}}\right]\right)+\ln \left(\mathbb{E}^{\mathbb{Q}}\left[\prod_{k=1}^{M} S_{k}(T)^{b_{k}}\right]\right)\right. \\
& \left.-(T-t)\left(\sum_{k=1}^{M} b_{k}\left(r-q_{k}-\frac{\sigma_{k}^{2}}{2}\right)-\sum_{k=M+1}^{M+N} b_{k}\left(r-q_{k}-\frac{\sigma_{k}^{2}}{2}\right)\right)\right) \text {. }
\end{aligned}
$$

with

$$
R(T) \equiv \frac{\prod_{k=1}^{M} S_{k}(T)^{b_{k}}}{\prod_{k=M+1}^{M+N} S_{k}(T)^{b_{k}}},
$$

and

$$
\sigma_{R}=\sqrt{\left\{\sum_{k=1}^{N+M} \sum_{l=1}^{N+M} \rho_{k l} \sigma_{k} \sigma_{l} m_{k} m_{l}\right\}}, \text { with } m_{j}= \begin{cases}F_{j} / \mathrm{e}^{\tilde{F}}, & \text { for } j=1, \cdots, M, \\ -F_{j} / \mathrm{e}^{\tilde{F}}, & \text { for } j=M+1, \cdots, M+N,\end{cases}
$$

where $\tilde{F}, \tilde{K}$ as well as the coefficients $\boldsymbol{b}=\left(b_{1}, \cdots, b_{M+N}\right)^{\mathrm{T}}$ are defined in Equations (24), (25) respectively, and where EBS stands for Extended Bjerksund and Stensland.

Proof. See Appendix A.2.

As far as the Greeks computation is concerned, this can be done in a straightforward 
way, since it is based on the calculation of the derivatives of the formula in Equation (26).

\section{Beyond the Black and Scholes Framework: Non-Gaussian Price Models}

In this section, we present two non-Gaussian price models on which we will analyze the performance of our approximation formula. For each model, we give a brief description and we provide the joint characteristic function of the assets log-returns $\phi_{T}(u)$ under the risk-neutral measure $\mathbb{Q}$.

\subsection{A Jump Diffusion Stock Model for the Equity Market}

In [32], a multivariate jump diffusion model is proposed in order to model asset prices in the equity market. In particular, the authors assume that the asset price $S(t)$ has two parts, a continuous part driven by a multivariate geometric Brownian motion, and a jump part with jump events modeled by a Poisson process. In the model, there are both common jumps and individual jumps. More precisely, if a Poisson event corresponds to a common jump, then all the asset prices will jump according to the multivariate asymmetric Laplace distribution; otherwise, if a Poisson event corresponds to an individual jump of the $j^{\text {th }}$ asset, then only the $j^{\text {th }}$ asset will jump. In other words, the model attempts to capture various ways of correlated jumps in asset prices.

Mathematically, under the risk-neutral measure $\mathbb{Q}$, the components of the stock price vector $S_{k}(t)$, for $k=1, \cdots, N+M$, have the following form:

$S_{k}(t)=S_{k}(0) \exp \left[\left(r-q_{k}-\frac{\sigma_{k}^{2}}{2}-\lambda \xi_{k}-\lambda_{k} v_{k}\right) t+\sigma_{k} W_{k}(t)+\sum_{m_{\mathrm{X}}=1}^{N_{k}(t)} X_{k}\left(m_{\mathrm{X}}\right)+\sum_{m_{\mathrm{Y}}=1}^{N(t)} Y_{k}\left(m_{\mathrm{Y}}\right)\right]$,

where $\sigma_{k}>0$, for $k=1, \cdots, N+M$, and $W_{i}, W_{j}$ are risk-neutral Brownian motions with instantaneous correlation $\rho_{i j},|\rho|<1$, for $i, j=1, \cdots, N+M$.

In addition,

$$
\sum_{m_{\mathrm{X}}=1}^{N_{k}(t)} X_{k}\left(m_{\mathrm{X}}\right), \quad \text { for } k=1, \cdots, N+M
$$

are $N+M$ univariate compound Poisson processes driven by the Poisson processes $N_{k}$ with intensity rate $\lambda_{k}$. As mentioned above, this jump component is unique to each stock and it describes the idiosyncratic shocks for that particular asset only. The idiosyncratic jump sizes $X_{k}$ are independently and identically distributed (i.i.d.) according to an asymmetric Laplace distribution $\mathcal{A} \mathcal{L} \mathcal{D}_{1}\left(m_{k}, v_{k}^{2}\right)$.

The Huang and Kou model in [32] also allows for common shocks described by

$$
\sum_{m_{\mathrm{Y}}=1}^{N(t)} Y\left(m_{\mathrm{Y}}\right)=\left(\sum_{m_{\mathrm{Y}}=1}^{N(t)} Y_{1}\left(m_{\mathrm{Y}}\right), \cdots, \sum_{m_{\mathrm{Y}}=1}^{N(t)} Y_{N+M}\left(m_{\mathrm{Y}}\right)\right)^{\mathrm{T}}
$$

which is a $(N+M)$-dimensional compound Poisson process with intensity rate $\lambda$.

Under the risk-neutral measure $\mathbb{Q}$, the jump sizes $Y$ are assumed to be independently and identically distributed according to a multivariate asymmetric Laplace distribution, 
$\mathcal{M} \mathcal{A L D}\left(\mathrm{a}, \Sigma_{\mathrm{Y}}\right)$, where $\mathrm{a}=\left(a_{1}, \cdots, a_{N+M}\right)^{\mathrm{T}}$ and $\Sigma_{\mathrm{Y}}$ is a $(N+M) \times(N+M)$ matrix, whose elements are defined as

$$
(\Sigma)_{k l}=\rho_{k l}^{\mathrm{Y}} \varepsilon_{k} \varepsilon_{l}, \quad \text { for } k=1, \cdots, N+M
$$

Finally, the quantities $\xi_{k}$ and $v_{k}$ in Equation (27) are defined respectively as:

$$
\xi_{k}=\frac{1}{1-a_{k}-\varepsilon_{k}^{2} / 2}-1, v_{k}=\frac{1}{1-m_{k}-v_{k}^{2} / 2}-1
$$

as reported in [26].

Then, the following proposition holds.

Proposition 4 (Caldana et al. (2016), Proposition 4). The joint characteristic function of the log-returns for the $(N+M)$-dimensional Huang and Kou jump diffusion model is given by:

$$
\begin{aligned}
\phi_{T}(\boldsymbol{u}) & =\exp \left(( T - t ) \left(i \boldsymbol{u}^{\mathrm{T}} \boldsymbol{s}-\frac{1}{2} \boldsymbol{u}^{\mathrm{T}} \Sigma \boldsymbol{u}+\frac{\lambda}{1-i \boldsymbol{u}^{\mathrm{T}} \boldsymbol{a}+\boldsymbol{u}^{\mathrm{T}} \Sigma_{\mathrm{Y}} \boldsymbol{u} / 2}-\lambda\right.\right. \\
& \left.\left.+\sum_{k=1}^{N+M}\left(\frac{\lambda_{k}}{1-i u_{k} m_{k}+u_{k}^{2} v_{k}^{2} / 2}-\lambda_{k}\right)\right)\right),
\end{aligned}
$$

where $(\Sigma)_{k l}=\rho_{k l} \sigma_{k} \sigma_{l}$ and $s_{k}=r-q_{k}-\sigma_{k}^{2} / 2-\lambda \xi_{k}-\lambda_{k} \varepsilon_{k}$, for $k=1, \cdots, N+M$.

Proof. As pointed out in [26], this follows from a straightforward generalization of the Huang and Kou model to the $(N+M)$-dimensional case.

\subsection{A Mean-Reverting Jump Diffusion Model for the Energy Market}

The second model we present here is a mean-reverting jump diffusion model discussed in [26] that generalizes the one proposed in [33] to describe the electricity spot price in the energy market.

As pointed out by the authors, a distinctive feature of electricity markets is the formation of price spikes which are caused when the maximum supply and current demand are close, often when a generator or part of the distribution network fails unexpectedly.

In particular, for $k=1, \cdots, N+M$, the spot price process $S_{k}(t)$ is defined to be the exponential of the sum of three components: a deterministic periodic function $f_{k}(t)$ characterising seasonality, an Ornstein-Uhlenbeck (OU) process $Y_{k}(t)$, and a meanreverting process with a jump component to incorporate spikes $X_{k}(t)$ :

$$
\left\{\begin{array}{l}
S_{k}(t)=\exp \left(f_{k}(t)+Y_{k}(t) X_{k}(t)\right), \\
\mathrm{d} Y_{k}(t)=-\omega_{k} Y_{k}(t) \mathrm{d} t+\sigma_{k} \mathrm{~d} W_{k}(t), \\
\mathrm{d} X_{k}(t)=-\omega_{k} X_{k}(t) \mathrm{d} t+J_{k}^{+} \mathrm{d} N_{k}^{+}(t)+J_{k}^{-} \mathrm{d} N_{k}^{-}(t),
\end{array}\right.
$$

where $\sigma_{k}>0$ and $W_{k}(t)$ is a risk-neutral Brownian motion.

As done in [26], we assume that the mean-reversion speed $\omega_{k}>0$ is the same for both the diffusion process $Y_{k}(t)$ and for the jump process $X_{k}(t)$. The Brownian mo- 
tions $W_{i}(t)$ and $W_{j}(t)$ have instantaneous correlation $\rho_{i j}$, with $\left|\rho_{i j}\right|<1$, for $i \neq j$ and equal to 1 for $i=j$.

$N_{k}^{+}(t)$ and $N_{k}^{-}(t)$ are Poisson processes with intensity $\lambda_{k}^{+}$and $\lambda_{k}^{-}$respectively, and they describe the positive and negative jump arrivals separately (this was not the case in the original model proposed by Hambly et al. in [33]). The jumps sizes $J_{k}^{+}$and $J_{k}^{-}$are assumed to be i.i.d. random variables exponentially distributed with parameters $0<\mu_{k}^{+}<1$ and $\mu_{k}^{-}>0$ respectively.

If we define the vector $z(t, T)$

$$
z_{k}(t, T) \equiv\left(Y_{k}(t)+X_{k}(t)\right)\left(\mathrm{e}^{-\omega_{k}(T-t)}-1\right)+f_{k}(T)-f_{k}(t),
$$

and the matrix $\Gamma(t, T)$

$$
\Gamma_{k l}(t, T) \equiv \rho_{k l} \frac{\sigma_{k} \sigma_{l}}{\omega_{k}+\omega l}\left(1-\mathrm{e}^{-\left(\omega_{k}+\omega_{l}\right)(T-t)}\right)
$$

and if we assume independence between the jump processes, then the following result holds.

Proposition 5 (Caldana et al. (2016), Proposition 5). The joint characteristic function of the log-returns for the $(N+M)$-dimensional mean-reverting jump diffusion model is given by:

$$
\begin{aligned}
\phi_{T}(\boldsymbol{u})= & \exp \left(i \boldsymbol{u}^{\mathrm{T}} \mathbf{z}(t, T)-\frac{1}{2} \boldsymbol{u}^{\mathrm{T}} \Gamma(t, T) \boldsymbol{u}+\sum_{k=1}^{N+M} \frac{\lambda_{k}^{+}}{\omega_{k}} \ln \left(\frac{1-i \mu_{k}^{+} u_{k} \mathrm{e}^{-\omega_{k}(T-t)}}{1-i \mu_{k}^{+} u_{k}}\right)\right. \\
& \left.+\sum_{k=1}^{N+M} \frac{\lambda_{k}^{-}}{\omega_{k}} \ln \left(\frac{1+i \mu_{k}^{-} u_{k} \mathrm{e}^{-\omega_{k}(T-t)}}{1+i \mu_{k}^{-} u_{k}}\right)\right) .
\end{aligned}
$$

Proof. As pointed out in [26], this follows from a generalization of the model in [33] to the $(N+M)$-dimensional case.

\section{Numerical Experiments}

In this section, we discuss numerical examples for the pricing of basket and multi-asset spread options under the assumption that the underlying prices follow the stochastic dynamics introduced in Sections 3 and 4.

\subsection{Geometric Brownian Motion Case}

\subsubsection{Basket Options}

In this section we deal with the pricing of basket options under the assumption of lognormality for the underlying asset prices. In particular, we compare our approximating formula in Equation (26) with the six different methods discussed in [34], namely:

- Levy (1992),

- Gentle (1993),

- Milevsky and Posner (1998a),

- Milevsky and Posner (1998b),

- Beisser (1999), and 
- Ju (2002)

As benchmark values, the authors in [34] consider a Monte Carlo simulation using antithetic method and geometric mean as control variate for variance reduction. The number of simulations was always chosen large enough to keep the standard deviation below 0.05 .

Input parameters are as in [34], where the authors focused on a call option on a basket with four stocks, whose weights are given by $\mathrm{w}=(0.25,0.25,0.25,0.25)$. More specifically, the model parameters are as follows: $T-t=5, r=0, S_{k}(t)=K=100$, $q_{k}=0, \sigma_{k}=0.40$, and $\rho_{k l}=0.50$, for $k, l=1, \cdots, 4$ and $k \neq l$, or 1 otherwise.

The authors in [34] looked at the influence of various parameters such as strike, correlations, underlying prices or volatilities on the performance of the different approximations. Their conclusion would suggest to use the method in [18] for homogeneous volatilities and the one in [13] for inhomogeneous ones. The switching rule is then the following: if the relative difference between the two computed values is less than $5 \%$ then use the price given in [18] for an upper bound, and the one given in [13] for a lower bound. If it is bigger than $5 \%$ then run a Monte Carlo simulation or if this is not suitable, keep the result given by the approach in [13].

In what follows, we will show that, using the same parameter setting, our approximating formula is as accurate as the best methods compared in [34]. For sake of completeness, we will compare it with the approaches discussed in [26], since the authors focused on the same numerical example. The results reported in Table 1 refer to the pricing of the basket option above for different strike prices $\mathrm{K}$, where $\mathrm{K}$ varies from 50 to 150. In particular, we rounded up to two decimals the results of our calculations in order to be consistent with the precision chosen in [34]. If we compare the results of the approximating formulas in Table 1, we can see that our formula has the same level of accuracy as the one in [26], with the advantage that no optimization routine has to be run $^{2}$. Besides that, our approximation is as accurate as the one proposed in [13]. As pointed out in Section 1, under the geometric Brownian motion case, the second approximating formula discussed in [26] coincides with the one in [15], which is less accurate than the other methods. We investigated the accuracy of our approximating formula by varying the input parameters as done in [34] and we found that our approximating formula has the same level of accuracy as the one considered in [13]. Results are available upon request.

\subsubsection{Multi-Asset Spread Options}

In this section we deal with the pricing of multi-asset spread options under the assumption of lognormality for the underlying asset prices. In particular, we compare our approximating formula in Equation (26) with the four different methods discussed in [35], namely:

- Improved Comonotonic Upper Bound (ICUB) as in Section 2.2 in [35],

${ }^{2} \mathrm{Up}$ to the fourth decimal, our formula coincides with the one proposed in [26]. This imply that our educated guess for the parameters $\tilde{F}, \tilde{K}$ and $\boldsymbol{b}$ turns out to be very efficient. 
Table 1. Prices of Basket Options (with $M=4, N=0$ ) computed for different strike prices $K$ in the GBM model of Section 3. Column EBS contains the Extended Bjerksund and Stensland pricing formula of Section 3.

\begin{tabular}{|c|c|c|c|c|c|c|c|c|}
\hline Spread & $K$ & Beisser (1999) & Gentle (1993) & $\mathrm{Ju}(2002)$ & Caldana et al. (2016) & EBS & $\mathrm{MC}$ & S.E. \\
\hline \multirow[t]{2}{*}{50} & 50 & 54.16 & 51.99 & 54.31 & 54.16 & 54.16 & 54.28 & 0.0383 \\
\hline & & $-0.22 \%$ & $-4.22 \%$ & $0.06 \%$ & $-0.22 \%$ & $-0.22 \%$ & & \\
\hline \multirow[t]{2}{*}{40} & 60 & 47.27 & 44.43 & 47.48 & 47.27 & 47.27 & 47.45 & 0.0375 \\
\hline & & $-0.38 \%$ & $-6.36 \%$ & $0.06 \%$ & $-0.38 \%$ & $-0.38 \%$ & & \\
\hline \multirow[t]{2}{*}{30} & 70 & 41.26 & 37.93 & 41.52 & 41.26 & 41.26 & 41.50 & 0.0369 \\
\hline & & $-0.58 \%$ & $-8.60 \%$ & $0.05 \%$ & $-0.58 \%$ & $-0.58 \%$ & & \\
\hline \multirow[t]{2}{*}{20} & 80 & 36.04 & 32.40 & 36.36 & 36.04 & 36.04 & 36.52 & 0.0363 \\
\hline & & $-1.31 \%$ & $-11.28 \%$ & $-0.44 \%$ & $-1.31 \%$ & $-1.31 \%$ & & \\
\hline \multirow[t]{2}{*}{10} & 90 & 31.53 & 27.73 & 31.88 & 31.53 & 31.53 & 31.85 & 0.0356 \\
\hline & & $-1.00 \%$ & $-12.94 \%$ & $0.09 \%$ & $-1.00 \%$ & $-1.00 \%$ & & \\
\hline \multirow[t]{2}{*}{0} & 100 & 27.63 & 23.78 & 28.01 & 27.63 & 27.63 & 27.98 & 0.0350 \\
\hline & & $-1.25 \%$ & $-15.01 \%$ & $0.11 \%$ & $-1.25 \%$ & $-1.25 \%$ & & \\
\hline \multirow[t]{2}{*}{-10} & 110 & 24.27 & 20.46 & 24.67 & 24.27 & 24.27 & 24.63 & 0.0344 \\
\hline & & $-1.46 \%$ & $-16.93 \%$ & $0.16 \%$ & $-1.46 \%$ & $-1.46 \%$ & & \\
\hline \multirow[t]{2}{*}{-20} & 120 & 21.36 & 17.65 & 21.77 & 21.36 & 21.36 & 21.74 & 0.0338 \\
\hline & & $-1.75 \%$ & $-18.81 \%$ & $0.14 \%$ & $-1.75 \%$ & $-1.75 \%$ & & \\
\hline \multirow[t]{2}{*}{-30} & 130 & 18.84 & 15.27 & 19.26 & 18.84 & 18.84 & 19.22 & 0.0332 \\
\hline & & $-1.98 \%$ & $-20.55 \%$ & $0.21 \%$ & $-1.98 \%$ & $-1.98 \%$ & & \\
\hline \multirow[t]{2}{*}{-40} & 140 & 16.65 & 13.25 & 17.07 & 16.65 & 16.65 & 17.05 & 0.0326 \\
\hline & & $-2.35 \%$ & $-22.29 \%$ & $0.12 \%$ & $-2.35 \%$ & $-2.35 \%$ & & \\
\hline \multirow[t]{2}{*}{-50} & 150 & 14.75 & 11.53 & 15.17 & 14.75 & 14.75 & 15.15 & 0.0320 \\
\hline & & $-2.64 \%$ & $-23.89 \%$ & $0.13 \%$ & $-2.64 \%$ & $-2.64 \%$ & & \\
\hline
\end{tabular}

- Shifted Log-Normal Approximation (SLN) as in [9],

- Hybrid Moment Matching with Improved Comonotonic Upper Bound (HMMICUB) as in Section 3.1 in [35] and

- Hybrid Moment Matching with Deng et al. (2008) Spread Approximation (HMMDLZ) as in Section 3.1 in [35].

As benchmark values, the authors in [35] consider a Monte Carlo simulation. Input parameters are as in [35], where the authors focused on spread call options written on three assets (see Table 4 and Table 7 in [35], whose weights are given by $\mathrm{w}=(1.0,-1.0,-1.0))$.

In what follows, we will show that, using the same parameter setting, our approximating formula outperforms the methods compared in [35]. For sake of completeness, we will compare it with the Extended Kirk formula proposed in [7] (see column labeled 
EK in Table 2, Table 3).

The results reported in Table 2, Table 3 refer to the pricing spread options for different strike prices $K$. In particular, in Table 2, the input parameters are as follows: $T-t=1, \quad r=0.05, \mathrm{~S}(t)=[100,24,46], \sigma=[0.40,0.22,0.30], \sigma=[0.40,0.22,0.30]$, $\rho_{13}=0.91, \rho_{13}=0.91$. For the results in Table 3 , the input parameters are as follows: $T-t=1, \quad T-t=1, \mathrm{~S}(t)=[100,63,12], \quad \sigma=[0.21,0.34,0.63], \quad \sigma=[0.21,0.34,0.63]$, $\sigma=[0.21,0.34,0.63], \rho_{23}=0.43$.

In both scenarios, our approximating formula outperforms the best methods considered in [35] as well as the approach in [7]. The approximation is less accurate for very deep out-of-the-money options, especially for the second case.

\subsection{Non-Gaussian Models}

In this section we deal with the pricing of basket and multi-asset spread options where the underlying asset prices follow the stochastic dynamics reported in Sections 4. In particular, we compare our approximating formula in Equation (6) with the the two approaches discussed in [26], namely:

- $C_{K}^{\mathcal{G}}(t)$, as in Equation (18),

- $C_{K}^{\mathrm{AG}}(t)$, based on the arithmetic-geometric average inequality, as in [26], Section 2.

Input parameters are as in [26]. For all the computations involving a Fourier inversion, we used a Gaussian quadrature rule, using Matlab's built-in function quadgk and a damping coefficient $\alpha=0.75$ as done in [26].

Table 2. Prices of Multi-Asset Spread Options (with $M=1, N=2$ ) computed for different strike prices $K$ in the GBM model of Section 3. Column EBS contains the Extended Bjerksund and Stensland pricing formula of Section 3. Number of simulations equal to 300 millions of paths and column label S.E. stands for Standard Error.

\begin{tabular}{cccccccccc}
\hline Spread & $K$ & ICUB & SLN & HMMICUB & HMMDLZ & EK & EBS & MC & S.E. \\
\hline 15 & 15 & 19.9819 & 19.6925 & 19.5251 & 19.5231 & 19.5848 & 19.6816 & 19.6849 & 0.0009 \\
& & $1.51 \%$ & $0.04 \%$ & $-0.81 \%$ & $-0.82 \%$ & $-0.51 \%$ & $-0.02 \%$ & & \\
10 & 20 & 17.0143 & 16.7345 & 16.5693 & 16.5673 & 16.6366 & 16.7009 & 16.7051 & 0.0008 \\
& & $1.85 \%$ & $0.18 \%$ & $-0.81 \%$ & $-0.82 \%$ & $-0.41 \%$ & $-\mathbf{0 . 0 3} \%$ & & \\
5 & 25 & 14.4105 & 14.1460 & 13.9964 & 13.9944 & 14.0731 & $\mathbf{1 4 . 0 9 6 1}$ & 14.1010 & 0.0008 \\
& & $2.19 \%$ & $0.32 \%$ & $-0.74 \%$ & $-0.76 \%$ & $-0.20 \%$ & $-0.03 \%$ & & \\
0 & 30 & 12.1523 & 11.9059 & 11.7811 & 11.7790 & 11.8687 & $\mathbf{1 1 . 8 4 6 6}$ & 11.8519 & 0.0007 \\
& & $2.53 \%$ & $0.46 \%$ & $-0.60 \%$ & $-0.62 \%$ & $0.14 \%$ & $-\mathbf{0 . 0 4 \%}$ & & \\
-5 & 35 & 10.2123 & 9.9851 & 9.8898 & 9.8876 & 9.9890 & $\mathbf{9 . 9 2 2 6}$ & 9.9281 & 0.0007 \\
& & $2.86 \%$ & $0.57 \%$ & $-0.39 \%$ & $-0.41 \%$ & $0.61 \%$ & $-\mathbf{0 . 0 6 \%}$ & & \\
-10 & 40 & 8.5588 & 8.3506 & 8.2860 & 8.2837 & 8.3962 & $\mathbf{8 . 2 8 9 7}$ & 8.2951 & 0.0006 \\
& & $3.18 \%$ & $0.67 \%$ & $-0.11 \%$ & $-0.14 \%$ & $1.22 \%$ & $-\mathbf{0 . 0 7 \%}$ & & \\
\hline & 45 & 7.1581 & 6.9683 & 6.9330 & 6.9305 & 7.0528 & $\mathbf{6 . 9 1 2 2}$ & 6.9174 & 0.0006 \\
& & $3.48 \%$ & $0.74 \%$ & $0.23 \%$ & $0.19 \%$ & $1.96 \%$ & $-\mathbf{0 . 0 7 \%}$ & & \\
\hline
\end{tabular}


Table 3. Prices of Multi-Asset Spread Options (with $M=1, N=2$ ) computed for different strike prices $K$ in the GBM model of Section 3. Column EBS contains the Extended Bjerksund and Stensland pricing formula of Section 3. Number of simulations equal to 15 millions of paths and column label S.E. stands for Standard Error.

\begin{tabular}{cccccccccc}
\hline Spread & $K$ & ICUB & SLN & HMMICUB & HMMDLZ & EK & EBS & MC & S.E. \\
\hline 22.50 & 2.50 & 24.6617 & 23.1681 & 23.5137 & 23.5138 & 23.4535 & 23.5493 & 23.5925 & 0.0009 \\
& & $4.53 \%$ & $-1.80 \%$ & $-0.33 \%$ & $-0.33 \%$ & $-0.59 \%$ & $-0.18 \%$ & & \\
15.00 & 10.00 & 18.5944 & 16.8591 & 17.1363 & 17.1373 & 17.0131 & 17.1596 & 17.2049 & 0.0008 \\
& & $8.08 \%$ & $-2.01 \%$ & $-0.40 \%$ & $-0.39 \%$ & $-1.11 \%$ & $-\mathbf{0 . 2 6 \%}$ & & \\
7.50 & 17.50 & 13.0945 & 11.3394 & 11.3854 & 11.3873 & 11.2434 & 11.3588 & 11.4099 & 0.0007 \\
& & $14.76 \%$ & $-0.62 \%$ & $-0.21 \%$ & $-0.20 \%$ & $-1.46 \%$ & $-0.45 \%$ & & \\
0 & 25.00 & 8.4135 & 6.9203 & 6.6579 & 6.6584 & 6.5548 & $\mathbf{6 . 5 4 1 8}$ & 6.6009 & 0.0006 \\
& & $27.46 \%$ & $4.84 \%$ & $0.86 \%$ & $0.87 \%$ & $-0.70 \%$ & $-\mathbf{0 . 8 9 \%}$ & & \\
-7.50 & 32.50 & 4.064 & 3.7629 & 3.3226 & 3.3147 & 3.2670 & $\mathbf{3 . 1 2 0 3}$ & 3.1872 & 0.0004 \\
& & $50.80 \%$ & $18.06 \%$ & $4.25 \%$ & $4.00 \%$ & $2.50 \%$ & $-\mathbf{2 . 1 0 \%}$ & & \\
-15.00 & 40.00 & 2.3929 & 1.7925 & 1.3950 & 1.3853 & 1.3636 & $\mathbf{1 . 1 8 5 2}$ & 1.2518 & 0.0003 \\
& & $91.16 \%$ & $43.19 \%$ & $11.44 \%$ & $10.66 \%$ & $8.93 \%$ & $-\mathbf{5 . 3 2 \%}$ & & \\
\hline
\end{tabular}

\subsubsection{Basket Options}

In Table 4 and Table 5, we report the results of our approximation formula when pricing basket options on 4 and 20 assets under the jump-diffusion model of Section 4.1 and the mean-reverting jump-diffusion model of Section 4.2 respectively.

As pointed out in [26], available methods in the literature which are based on the knowledge of the characteristic function, such as [29] [36] [37] and [38], suffer from the curse of dimensionality, as they require an M-dimensional quadrature and therefore they cannot be used in practice when the basket dimension is high. Besides that, the first three methods require assumptions on the form of the characteristic function that rule out mean-reverting models, therefore not applicable even to the mean-reverting jump-diffusion model considered in Section 4.2.

In order to deal with the curse of dimensionality, we are aware of a method discussed in [39], where the authors propose a parallel partitioning approach. However, they do not provide results for basket options with dimension grater than seven.

If we look at Table 4, we can see that our approximating formula gives very close results to the one proposed by [26]. It seems that also for the jump-diffusion model of Section 4.1, the educated guess proposed in Section 2 turns out to be very efficient as it avoids us from running an optimization routine. The second approach in [26] produces less accurate results than our approximation. As observed in the geometric Brownian motion case, our approximating formula is less accurate for deep out-of-the-money options. 
Table 4. Prices of Basket Options (with $M=20, N=0$ ) computed for different strikes $K$ in the JD model of Section 4.1. The basket weights are $\mathbf{w}=\frac{1}{20} \mathbf{1}_{20}$. The model parameters are: $T-t=1$, $r=0.01, \quad S_{k}(t)=100, \quad \sigma_{k}=0.40, \quad \epsilon_{k}=0.5, \quad v_{k}=0.30, \quad a_{k}=m_{k}=-0.05, \lambda=1, \quad \lambda_{k}=0.5$, $\rho_{k l}=0.5$ and $\rho_{k l}^{\mathrm{Y}}=0.5$ for $k, l=1, \cdots, 20$ and $k \neq l, 1$ otherwise. Column ECF contains the Extended Caldana and Fusai pricing formula of Section 2. Columns MC and C.I. Length contain the Monte Carlo option price and 95\% confidence interval. The Monte Carlo price is obtained with 1000000 simulations, see [26].

\begin{tabular}{|c|c|c|c|c|c|c|}
\hline Spread & $K$ & $C_{K}^{\mathcal{G}}(t)$ & $C_{K}^{\mathrm{AG}}(t)$ & ECF & MC & C.I. Length \\
\hline \multirow[t]{2}{*}{50} & 50 & 51.3843 & 51.0643 & 51.3769 & 51.4609 & $3.6686 \times 10^{-3}$ \\
\hline & & $-0.15 \%$ & $-0.77 \%$ & $-0.16 \%$ & & \\
\hline \multirow[t]{2}{*}{40} & 60 & 42.6134 & 41.9998 & 42.6132 & 42.7487 & $5.2246 \times 10^{-3}$ \\
\hline & & $-0.32 \%$ & $-1.75 \%$ & $-0.32 \%$ & & \\
\hline \multirow[t]{2}{*}{30} & 70 & 34.7380 & 33.6724 & 34.7329 & 34.9309 & $6.5806 \times 10^{-3}$ \\
\hline & & $-0.55 \%$ & $-3.60 \%$ & $-0.57 \%$ & & \\
\hline \multirow[t]{2}{*}{20} & 80 & 27.9956 & 26.3980 & 27.9769 & 28.2380 & $7.9691 \times 10^{-3}$ \\
\hline & & $-0.86 \%$ & $-6.52 \%$ & $-0.92 \%$ & & \\
\hline \multirow[t]{2}{*}{10} & 90 & 22.4645 & 20.3784 & 22.4336 & 22.7460 & $9.1516 \times 10^{-3}$ \\
\hline & & $-1.24 \%$ & $-10.41 \%$ & $-1.37 \%$ & & \\
\hline \multirow[t]{2}{*}{0} & 100 & 18.0726 & 15.6289 & 18.0371 & 18.3801 & $1.0277 \times 10^{-2}$ \\
\hline & & $-1.67 \%$ & $-14.97 \%$ & $-1.87 \%$ & & \\
\hline \multirow[t]{2}{*}{-10} & 110 & 14.6576 & 12.0121 & 14.6257 & 14.9774 & $1.1022 \times 10^{-2}$ \\
\hline & & $-2.14 \%$ & $-19.80 \%$ & $-2.35 \%$ & & \\
\hline \multirow[t]{2}{*}{-20} & 120 & 12.0282 & 9.3174 & 12.0047 & 12.3481 & $1.1549 \times 10^{-2}$ \\
\hline & & $-2.59 \%$ & $-24.54 \%$ & $-2.78 \%$ & & \\
\hline \multirow[t]{2}{*}{-30} & 130 & 10.0046 & 7.3281 & 9.9907 & 10.3229 & $1.2029 \times 10^{-2}$ \\
\hline & & $-3.08 \%$ & $-29.01 \%$ & $-3.22 \%$ & & \\
\hline \multirow[t]{2}{*}{-40} & 140 & 8.4370 & 5.8579 & 8.4310 & 8.7468 & $1.2316 \times 10^{-2}$ \\
\hline & & $-3.54 \%$ & $-33.03 \%$ & $-3.61 \%$ & & \\
\hline \multirow[t]{2}{*}{-50} & 150 & 7.2090 & 4.7614 & 7.2076 & 7.5032 & $1.2477 \times 10^{-2}$ \\
\hline & & $-3.92 \%$ & $-36.54 \%$ & $-3.94 \%$ & & \\
\hline
\end{tabular}


Table 5. Prices of Basket Options (with $M=4, N=0$ ) computed for different strikes $K$ in the MRJD model of Section 4.2. The basket weights are $\mathbf{w}=(0.25,0.25,0.25,0.25)$. The model parameters are: $T-t=1, r=0, f_{k}(t)=\ln (25), \omega=\left(\omega_{k}\right)_{k}=(0.1,0.2,0.1,0.3), \quad Y_{k}(t)=X_{k}(t)=0$ for $k=1, \cdots, 4$. Jump parameters are $\lambda^{+}=\lambda^{-}=(0.1,0.2,0.3,0.2)$ and $\mu^{+}=\mu^{-}=(0.1,0.1,0.3,0.3)$. The covariance matrix for the Brownian part is equal to $(0.5,0.35,0.35,0.25,0.35 ; 0.35,0.50$, $0.475,0.15 ; 0.35,0.475,0.50,0.15 ; 0.25,0.15,0.15,0.50)$. Column ECF contains the Extended Caldana and Fusai pricing formula of Section 2. The Monte Carlo price is obtained with 100000 simulations and 100 time steps, see [26]. Columns labels are the same as in Table 4.

\begin{tabular}{|c|c|c|c|c|c|c|}
\hline Spread & $K$ & $C_{K}^{\mathcal{G}}(t)$ & $C_{K}^{\mathrm{AG}}(t)$ & ECF & MC & C.I. Length \\
\hline \multirow[t]{2}{*}{20} & 5 & 26.5940 & 26.5936 & 26.5910 & 26.5942 & $1.4267 \times 10^{-4}$ \\
\hline & & $0.00 \%$ & $0.00 \%$ & $-0.01 \%$ & & \\
\hline \multirow[t]{2}{*}{15} & 10 & 21.6486 & 21.6104 & 21.5761 & 21.6547 & $1.4270 \times 10^{-3}$ \\
\hline & & $-0.03 \%$ & $-0.20 \%$ & $-0.36 \%$ & & \\
\hline \multirow[t]{2}{*}{10} & 15 & 17.0447 & 16.8630 & 16.8108 & 17.0682 & $3.3615 \times 10^{-3}$ \\
\hline & & $-0.14 \%$ & $-1.20 \%$ & $-1.51 \%$ & & \\
\hline \multirow[t]{2}{*}{5} & 20 & 13.1066 & 12.7435 & 12.7100 & 13.1508 & $3.3615 \times 10^{-3}$ \\
\hline & & $-0.34 \%$ & $-3.10 \%$ & $-3.35 \%$ & & \\
\hline \multirow[t]{2}{*}{0} & 25 & 9.9462 & 9.4451 & 9.4431 & 10.0063 & $6.6941 \times 10^{-3}$ \\
\hline & & $-0.60 \%$ & $-5.61 \%$ & $-5.63 \%$ & & \\
\hline \multirow[t]{2}{*}{-5} & 30 & 7.5059 & 6.9338 & 6.9588 & 7.5795 & $8.5450 \times 10^{-3}$ \\
\hline & & $-0.97 \%$ & $-8.52 \%$ & $-8.19 \%$ & & \\
\hline \multirow[t]{2}{*}{-10} & 35 & 5.6608 & 5.0748 & 5.1170 & 5.7401 & $9.4264 \times 10^{-3}$ \\
\hline & & $-1.38 \%$ & $-11.59 \%$ & $-10.86 \%$ & & \\
\hline \multirow[t]{2}{*}{-15} & 40 & 4.2798 & 3.7181 & 3.7688 & 4.3585 & $1.0472 \times 10^{-2}$ \\
\hline & & $-1.81 \%$ & $-14.69 \%$ & $-13.53 \%$ & & \\
\hline \multirow[t]{2}{*}{-20} & 45 & 3.2497 & 2.7337 & 2.7865 & 3.3310 & $1.1202 \times 10^{-2}$ \\
\hline & & $-2.44 \%$ & $-17.93 \%$ & $-16.35 \%$ & & \\
\hline
\end{tabular}

Results for the mean-reverting jump-diffusion model in Table 5 show that our approximating formula is outperformed by the first approach in [26]. In this case, it seems that an optimization routine for the optimal parameters is required to produce better results. However, the accuracy of our approximating formula is still higher than the second approach in [26] and the approximation is still accurate for in-the-money options.

\subsubsection{Multi-Asset Spread Options}

In Table 6 and Table 7 we deal with the pricing of multi-asset spread options under the 
Table 6. Prices of Multi-Asset Spread Options (with $M=4, N=0$ ) computed for different strikes $K$ in the JD model of Section 4.1. The weights are $\mathrm{w}=(1.0,1.0,1.0,1.0,1.0,1.0,1.0,1.0,1.0,1.0$, $-0.90,-0.90,-0.90,-0.90,-0.90,-0.90,-0.90,-0.90,-0.90,-0.90)$. The model parameters are: $T$ - $t=1, r=0.01, S_{k}(t)=100, \sigma_{k}=0.40, \epsilon_{k}=0.25, \quad v_{k}=0.15, a_{k}=m_{k}=-0.05, \lambda=1$, $\lambda_{k}=0.1, \rho_{k l}=0.75$ and $\rho_{k l}^{\mathrm{Y}}=0.75$ for $k, l=1, \cdots, 20$ and $k \neq l, 1$ otherwise. Column ECF contains the Extended Caldana and Fusai pricing formula of Section 2. Columns $M C$ and C.I. Length contain the Monte Carlo option price and 95\% confidence interval. The Monte Carlo price is obtained with 1000000 simulations, see [26].

\begin{tabular}{|c|c|c|c|c|c|c|}
\hline Spread & $K$ & $C_{K}^{\mathcal{G}}(t)$ & $C_{K}^{\mathrm{AG}}(t)$ & ECF & MC & C.I. Length \\
\hline \multirow[t]{2}{*}{50} & 50 & 59.7381 & 59.9191 & 60.0094 & 60.2354 & $1.7226 \times 10^{-2}$ \\
\hline & & $-0.83 \%$ & $-0.53 \%$ & $-0.38 \%$ & & \\
\hline \multirow[t]{2}{*}{40} & 60 & 52.4154 & 52.4967 & 52.5980 & 52.8421 & $1.5666 \times 10^{-2}$ \\
\hline & & $-0.81 \%$ & $-0.65 \%$ & $-0.46 \%$ & & \\
\hline \multirow[t]{2}{*}{30} & 70 & 45.6244 & 45.6139 & 45.7291 & 45.9875 & $1.4105 \times 10^{-2}$ \\
\hline & & $-0.79 \%$ & $-0.81 \%$ & $-0.56 \%$ & & \\
\hline \multirow[t]{2}{*}{20} & 80 & 39.4076 & 39.3213 & 39.4528 & 39.7336 & $1.3311 \times 10^{-2}$ \\
\hline & & $-0.82 \%$ & $-1.04 \%$ & $-0.71 \%$ & & \\
\hline \multirow[t]{2}{*}{10} & 90 & 33.7918 & 33.6515 & 33.8009 & 34.0946 & $1.2744 \times 10^{-2}$ \\
\hline & & $-0.89 \%$ & $-1.30 \%$ & $-0.86 \%$ & & \\
\hline \multirow[t]{2}{*}{0} & 100 & 28.7861 & 28.6166 & 28.7845 & 29.0848 & $1.2179 \times 10^{-2}$ \\
\hline & & $-1.03 \%$ & $-1.61 \%$ & $-1.03 \%$ & & \\
\hline \multirow[t]{2}{*}{-10} & 110 & 24.3817 & 24.2075 & 24.3932 & 24.6916 & $1.2049 \times 10^{-2}$ \\
\hline & & $-1.26 \%$ & $-1.96 \%$ & $-1.21 \%$ & & \\
\hline \multirow[t]{2}{*}{-20} & 120 & 20.5538 & 20.3964 & 20.5980 & 20.8951 & $1.2273 \times 10^{-2}$ \\
\hline & & $-1.63 \%$ & $-2.39 \%$ & $-1.42 \%$ & & \\
\hline \multirow[t]{2}{*}{-30} & 130 & 17.2644 & 17.1400 & 17.3549 & 17.6510 & $1.2885 \times 10^{-2}$ \\
\hline & & $-2.19 \%$ & $-2.90 \%$ & $-1.68 \%$ & & \\
\hline \multirow[t]{2}{*}{-40} & 140 & 14.4663 & 14.3852 & 14.6100 & 14.8944 & $1.3328 \times 10^{-2}$ \\
\hline & & $-2.87 \%$ & $-3.42 \%$ & $-1.91 \%$ & & \\
\hline \multirow[t]{2}{*}{-50} & 150 & 12.1067 & 12.0735 & 12.3047 & 12.5775 & $1.4203 \times 10^{-2}$ \\
\hline & & $-3.74 \%$ & $-4.01 \%$ & $-2.17 \%$ & & \\
\hline
\end{tabular}


Table 7. Prices of Multi-Asset Spread Options (with $M=2, N=2$ ), computed for different strikes $K$ in the MRJD model of Section 4.2. The weights are $w=(2,1,-1,-1)$. The model parameters are: $T-t=1, r=0, f_{k}(t)=\ln (25), \omega=\left(\omega_{k}\right)_{k}=(0.1,0.2,0.1,0.3), \quad Y_{k}(t)=X_{k}(t)=0$ for $k=1$, ..., 4. Jump parameters are $\lambda^{+}=\lambda^{-}=(0.1,0.2,0.3,0.2)$ and $\mu^{+}=\mu^{-}=(0.1,0.1,0.3,0.3)$. The covariance matrix for the Brownian part is equal to $(0.5,0.35,0.35,0.25,0.35 ; 0.35,0.50,0.475$, 0.15; 0.35. 0.475. 0.50. 0.15; 0.25, 0.15, 0.15, 0.50). Column ECF contains the Extended Caldana and Fusai pricing formula of Section 2. The Monte Carlo price is obtained with 100000 simulations and 100 time steps, see [26]. Columns labels are the same as in Table 6.

\begin{tabular}{|c|c|c|c|c|c|c|}
\hline Spread & $K$ & $C_{K}^{\mathcal{G}}(t)$ & $C_{K}^{\mathrm{AG}}(t)$ & ECF & MC & C.I. Length \\
\hline \multirow[t]{2}{*}{20} & 5 & 27.7176 & 27.4510 & 29.0320 & 28.6779 & $1.0476 \times 10^{-1}$ \\
\hline & & $-3.35 \%$ & $-4.28 \%$ & $1.23 \%$ & & \\
\hline \multirow[t]{2}{*}{15} & 10 & 24.2968 & 23.9533 & 25.4614 & 25.0422 & $8.5187 \times 10^{-2}$ \\
\hline & & $-2.98 \%$ & $-4.35 \%$ & $1.67 \%$ & & \\
\hline \multirow[t]{2}{*}{10} & 15 & 21.2185 & 20.8709 & 22.2513 & 21.8059 & $7.1737 \times 10^{-2}$ \\
\hline & & $-2.69 \%$ & $-4.29 \%$ & $2.04 \%$ & & \\
\hline \multirow[t]{2}{*}{5} & 20 & 18.4747 & 18.1838 & 19.4079 & 18.9539 & $6.4985 \times 10^{-2}$ \\
\hline & & $-2.53 \%$ & $-4.06 \%$ & $2.40 \%$ & & \\
\hline \multirow[t]{2}{*}{0} & 25 & 16.0482 & 15.8554 & 16.9166 & 16.4551 & $5.6703 \times 10^{-2}$ \\
\hline & & $-2.47 \%$ & $-3.64 \%$ & $2.80 \%$ & & \\
\hline \multirow[t]{2}{*}{-5} & 30 & 13.9159 & 13.8434 & 14.7496 & 14.3020 & $5.2007 \times 10^{-2}$ \\
\hline & & $-2.70 \%$ & $-3.21 \%$ & $3.13 \%$ & & \\
\hline \multirow[t]{2}{*}{-10} & 35 & 12.0514 & 12.1065 & 12.8735 & 12.4293 & $4.8954 \times 10^{-2}$ \\
\hline & & $-3.04 \%$ & $-2.60 \%$ & $3.57 \%$ & & \\
\hline \multirow[t]{2}{*}{-15} & 40 & 10.4277 & 10.6068 & 11.2534 & 10.8309 & $4.5859 \times 10^{-2}$ \\
\hline & & $-3.72 \%$ & $-2.07 \%$ & $3.90 \%$ & & \\
\hline \multirow[t]{2}{*}{-20} & 45 & 9.0178 & 9.3106 & 9.8558 & 9.4376 & $4.3508 \times 10^{-2}$ \\
\hline & & $-4.45 \%$ & $-1.35 \%$ & $4.43 \%$ & & \\
\hline
\end{tabular}

non-Gaussian models of Section 4. If we look at the results in Table 6, for the jumpdiffusion model, we can see that our approximation outperforms both approaches in [26]. Once again, it seems that the educated guess proposed in Section 2 produces very accurate results and avoids us from running an optimization routine. The accuracy of the approximations decreases when passing from in-the-money options to very deep out-of-the-money options.

Results in Table 7 for the mean-reverting jump-diffusion model show that for very deep in-the-money options, our approximating formula produces very accurate results 
compared to the approaches in [26], but in this case the educated guess of Section 2 produces an upper bound for the fair price given by Monte Carlo simulation. For at-the-money and out-of-the-money options, the second approach in [26] seems to produce a better approximation to the option price. However, the approximation error of our formula compared to the Monte Carlo simulation is within $1 \%-4 \%$.

\section{Conclusions}

This paper presents an approximating formula for the pricing of basket and multi-asset spread options, which genuinely extends the one in [25] for two-asset spread options. The technique we propose is applicable whenever the joint characteristic function of the vector of log-returns is known. We test our approximating formula on different multivariate models, allowing for jumps and nothing prevents the reader from applying our approach also under a stochastic volatility framework. Besides, under the lognormal setting, we show that our formula becomes a Black and Scholes type formula, extending the formula in [30]. Numerical experiments and comparison with Monte Carlo simulations and other methods available in the literature are discussed. The main contribution of this paper is to provide practitioners with a pricing formula, which can be used for real-time pricing of basket and multi-asset spread options, even under a nonGaussian framework.

\section{References}

[1] Black, F. and Scholes, M. (1973) The Pricing of Option and Corporate Liabilities. Journal of Political Economy, 81, 637-654. https://doi.org/10.1086/260062

[2] Carmona, R. and Durrleman, V. (2003) Pricing and Hedging Spread Options in a Lognormal Model. Technical Report, Princeton OPFE.

[3] Carmona, R. and Durrleman, V. (2006) Generalizing the Black-Scholes Formula to Multivariate Contingent Claims. Journal of Computational Finance, 9, 43-67.

https://doi.org/10.21314/JCF.2005.159

[4] Deng, S.J., Li, M. and Zhou, J. (2010) Multi-Asset Spread Option Pricing and Hedging. Quantitative Finance, 10, 305-324.

[5] Kirk, E. (1995) Correlations in the Energy Markets. In: Kaminsk, V., Ed., Managing Energy Price Risk, Risk Publications, London, 71-78.

[6] Alòs, E., Eydeland, A. and Laurence, P. (2011) A Kirk's and a Bachelier's Formula for Three-Asset Spread Options. Energy Risk, 9, 52-57.

[7] Lau, C.S. and Lo, C.F. (2014) The Pricing of Basket-Spread Options. Quantitative Finance, 14, 1971-1982. https://doi.org/10.1080/14697688.2014.949289

[8] Deng, S.J., Li, M. and Zhou, J. (2008) Closed-Form Approximations for Spread Option Prices and Greeks. Journal of Derivatives, 15, 58-80.

[9] Borovkova, S., Permana, F.J. and Weide, H. (2007) A Closed-Form Approach to the Valuation and Hedging of Basket and Spread Options. Journal of Derivatives, 14, 8-24. https://doi.org/10.3905/jod.2007.686420

[10] Pellegrino, T. and Sabino, P. (2014) On the Use of the Moment-Matching Technique for Pricing and Hedging Multi-Asset Spread Options. Energy Economics, 45, 172-185. https://doi.org/10.1016/j.eneco.2014.06.014 
[11] Pellegrino, T. andSabino, P. (2014) Pricing and Hedging Multi-Asset Spread Options using a Three-Dimensional Fourier Cosine Series Expansion Method. Journal of Energy Markets, 7, 71-92. https://doi.org/10.21314/JEM.2014.117

[12] Curran, M. (1994) Valuing Asian and Portfolio Options by Conditioning on the Geometric Mean Price. Management Science, 40, 1705-1711. https://doi.org/10.1287/mnsc.40.12.1705

[13] Beisser, J. (1999) Another Way to Value Basket Options. Working paper, Johannes Gutenberg-Universitt, Mainz.

[14] Rogers, L.C.G. and Shi, Z. (1995) The value of an Asian Option. Journal of Applied Probability, 32, 1077-1088. https://doi.org/10.1017/S0021900200103559

[15] Gentle, D. (1993) Basket Weaving. Risk, 6, 51-52.

[16] Vorst, T. (1992) Prices and Hedges of Average Exchange Rate Options. International Review of Financial Analysis, 1, 179-193. https://doi.org/10.1016/1057-5219(92)90003-M

[17] Levy, E. (1992) Pricing European Average Rate Currency Options. Journal of International Money and Finance, 11, 474-491. https://doi.org/10.1016/0261-5606(92)90013-N

[18] Ju, E. (2002) Pricing Asian and Basket Options via Taylor Expansion. Journal of Computational Finance, 5, 79-103. https://doi.org/10.21314/JCF.2002.088

[19] Milevsky, M.A. and Posner, S.E. (1998) A Closed-Form Approximation for Valuing Basket Options. Journal of Derivatives, 5, 54-61. https://doi.org/10.3905/jod.1998.408005

[20] Milevsky, M.A. and Posner, S.E. (1998) Valuing Exotic Options by Approximating the SPD with Higher Moments. Journal of Financial Engineering, 7, 109-125.

[21] Johnson, N.L. (1949) Systems of Frequency Curves Generated by Methods of Translation. Biometrika, 36, 149-176. https://doi.org/10.1093/biomet/36.1-2.149

[22] Deelstra, G., Liinev, J. and Vanmaele, M. (2004) Pricing of Arithmetic Basket Optionsby Conditioning. Insurance: Mathematics and Economics, 31, 55-77. https://doi.org/10.1016/j.insmatheco.2003.11.002

[23] Vanmaele, M., Deelstra, G. andLiinev, G. (2004) Approximation of Stop-Loss Premiums Involving Sums of Lognormals by Conditioning on Two Variables. Insurance: Mathematics and Economics, 35, 343-367. https://doi.org/10.1016/j.insmatheco.2004.06.001

[24] Deelstra, G., Diallo, I. and Vanmaele, M. (2008) Bounds for Asian Basket Options. Journal of Computational and Applied Mathematics, 218, 215-228. https://doi.org/10.1016/j.cam.2006.12.017

[25] Caldana, R. and Fusai, G. (2013) A General Closed-Form Spread Option Pricing Formula. Journal of Banking and Finance, 37, 4893-4906. https://doi.org/10.1016/j.jbankfin.2013.08.016

[26] Caldana, R., Fusai, G., Gnoatto, A. and Grasselli, M. (2016) General Closed-Form Basket Option Pricing Bounds. Quantitative Finance, 16, 535-554. https://doi.org/10.1080/14697688.2015.1073854

[27] Dempster, M.A.H. and Hong, S.S.G. (2002) Spread Option Valuation and the Fast Fourier Transform. In: Geman, H., Madan, D., Pliska, S.R. and Vorst, T., Eds., Mathetmacial Finance-Bachelier Congress 2000, Springer, Berlin, 203-220. https://doi.org/10.1007/978-3-662-12429-1_10

[28] Carr, P. and Madan, D. (2000) Option Valuation using the Fast Fourier Transform. Journal of Computational Finance, 2, 61-73. https://doi.org/10.21314/JCF.1999.043

[29] Hurd, T.R. and Zhou, Z. (2010) A Fourier Transform Method for Spread Options Pricing. SIAM Journal of Financial Mathematics, 1, 142-157. https://doi.org/10.1137/090750421 
[30] Bjerksund, P. and Stensland, G. (2011) Closed Form Spread Option Valuation. Quantitative Finance, 14, 1785-1794.

[31] Green, R. (2015) Closed Form Valuation of Three-Asset Spread Options With a View towards Clean Dark Spreads. Knut Wicksell, Working Paper.

[32] Huang, Z. and Kou, S.G. (2006) First Passage Times and Analytical Solutions for Options on Two Assets with Jump Risk. Preprint, Columbia University.

[33] Hambly, B. Howinson, S. and Kluge, T. (2009) Modelling Spikes and Pricing Swing Options in Electricity Markets. Quantitative Finance, 9, 937-949.

https://doi.org/10.1080/14697680802596856

[34] Krekel, M., De Kock, J., Korn, R. and Man, T.K. (2004) An Analysis of Pricing Methods for Basket Options. Wilmott, 3, 82-89. https://doi.org/10.1002/wilm.42820040318

[35] Deelstra, G., Petkovic, A. and Vanmaele, M. (2010) Pricing and Hedging Asian Basket Spread Options. Journal of Computational and Applied Mathematics, 234, 2814-2830. https://doi.org/10.1016/j.cam.2009.11.027

[36] Jackson, K.R., Jaimungal, S. and Surkov, V. (2008) Fourier Space Time-Stepping for Option Pricing with Levy Models. The Journal of Computational Finance, 12, 1-29. https://doi.org/10.21314/JCF.2008.178

[37] Lord, R., Fang, F., Bervoets, F. and Oosterlee, C.W. (2008) A fast and Accurate FFT-based Method for Pricing Early-Exercise Options under Levy Processes. SIAM Journal on Scientific Computing, 30, 1678-1705. https://doi.org/10.1137/070683878

[38] Jaimungal, S. and Surkov, V. (2011) Levy based Cross-Commodity Models and Derivative Valuation. SIAM Journal of Financial Mathematics, 2, 464-487. https://doi.org/10.1137/100791609

[39] Leentvaar, C.C.W. and Oosterlee, C.W. (2008) Multi-Asset Option Pricing Using a Parallel Fourier-based Technique. The Journal of Computational Finance, 12, 1-26. https://doi.org/10.21314/JCF.2008.184

[40] Johnson, R.A. and Wichern, D.W. (2002) Applied Multivariate Statistical Analysis. Prentice Hall, New Jersey. 


\section{Appendix}

\section{A.1. Proof of Proposition 1}

Proof. We observe that

$$
\begin{aligned}
\mathbb{E}^{\mathbb{Q}}\left[\prod_{l=1}^{M} S_{l}(T)^{b_{l}}\right] & =\mathbb{E}^{\mathbb{Q}}\left[\exp \left(\sum_{l=1}^{M} b_{l} \ln S_{l}(T)\right)\right]=\mathbb{E}^{\mathbb{Q}}\left[\exp \left(\sum_{l=1}^{M} b_{l} X_{l}(T)\right)\right] \\
& =\phi_{T}\left(u_{1}=-i b_{1}, \cdots, u_{M}=-i b_{M}, u_{M+1}=0, \cdots, u_{M+N}=0\right),
\end{aligned}
$$

and the same holds for

$$
\begin{aligned}
\mathbb{E}^{\mathbb{Q}}\left[\prod_{l=M+1}^{M+N} S_{l}(T)^{b_{l}}\right] & =\mathbb{E}^{\mathbb{Q}}\left[\exp \left(\sum_{l=M+1}^{M+N} b_{l} \ln S_{l}(T)\right)\right]=\mathbb{E}^{\mathbb{Q}}\left[\exp \left(\sum_{l=M+1}^{M+N} b_{l} X_{l}(T)\right)\right] \\
& =\phi_{T}\left(u_{1}=0, \cdots, u_{M}=0, u_{M+1}=-i b_{M+1}, \cdots, u_{M+N}=-i b_{M+N}\right) .
\end{aligned}
$$

Therefore, we can re-write the set $A$ defined in Equation (2) as

$$
\begin{aligned}
A= & \left\{\omega: \sum_{l=1}^{M} b_{l} X_{l}(T)-\sum_{l=M+1}^{M+N} b_{l} X_{l}(T)>\tilde{K}-\tilde{F}\right. \\
& +\ln \phi_{T}\left(u_{1}=-i b_{1}, \cdots, u_{M}=-i b_{M}, u_{M+1}=0, \cdots, u_{M+N}=0\right) \\
& \left.-\ln \phi_{T}\left(u_{1}=0, \cdots, u_{M}=0, u_{M+1}=-i b_{M+1}, \cdots, u_{M+N}=-i b_{M+N}\right)\right\}
\end{aligned}
$$

i.e.

$$
\begin{aligned}
A= & \left\{\omega: \tilde{K}<\sum_{l=1}^{M} b_{l} X_{l}(T)-\sum_{l=M+1}^{M+N} b_{l} X_{l}(T)+\tilde{F}\right. \\
& -\ln \phi_{T}\left(u_{1}=-i b_{1}, \cdots, u_{M}=-i b_{M}, u_{M+1}=0, \cdots, u_{M+N}=0\right) \\
& \left.+\ln \phi_{T}\left(u_{1}=0, \cdots, u_{M}=0, u_{M+1}=-i b_{M+1}, \cdots, u_{M+N}=-i b_{M+N}\right)\right\} .
\end{aligned}
$$

Following [27] [28] and [25], we multiply the expected value of the multi-asset spread call option approximation

$$
\mathbb{E}^{\mathbb{Q}}\left[\left(\sum_{l=1}^{M} w_{l} S_{l}(T)-\sum_{l=M+1}^{M+N} w_{l} S_{l}(T)-K\right)_{+} \nVdash_{\{A\}}\right]
$$

by an exponentially decaying term, tuned by a parameter $\alpha$, such that it is square integrable in $\tilde{K}$ over the negative axis.

Then, we apply the Fourier transform to this modified call option price for the multi-asset spread option pricing problem, as follows:

$$
\begin{aligned}
\Psi_{T}(\gamma ; \alpha, \tilde{F}, \tilde{K}, \mathbf{b}) & =\int_{\mathbb{R}} \mathrm{e}^{i \gamma \tilde{K}+\alpha \tilde{K}} \mathbb{E}^{\mathbb{Q}}\left[\left(\sum_{l=1}^{M} w_{l} S_{l}(T)-\sum_{l=M+1}^{M+N} w_{l} S_{l}(T)-K\right)_{+} \nVdash_{\{A\}}\right] \mathrm{d} \tilde{K} \\
& =\int_{\mathbb{R}}\left[\mathrm{e}^{i \gamma \tilde{K}+\alpha \tilde{K}} \int_{\mathbb{R}^{M+N}}\left(\sum_{l=1}^{M} w_{l} \mathrm{e}^{X_{l}(T)}-\sum_{l=M+1}^{M+N} w_{l} \mathrm{e}^{X_{l}(T)}-K\right) \nVdash_{\{A\}} f(\mathbf{X}) \mathrm{d} \mathbf{X}\right] \mathrm{d} \tilde{K} .
\end{aligned}
$$

Now, from Equation (31) and by applying Fubini's theorem, we can re-write the above expression as

$$
\Psi_{T}(\gamma ; \alpha, \tilde{F}, \tilde{K}, \mathbf{b})=\int_{\mathbb{R}^{M+N}}\left[\int_{-\infty}^{h} \mathrm{e}^{i \gamma \tilde{K}+\alpha \tilde{K}} \mathrm{~d} \tilde{K}\right]\left(\sum_{l=1}^{M} w_{l} \mathrm{e}^{X_{l}(T)}-\sum_{l=M+1}^{M+N} w_{l} \mathrm{e}^{X_{l}(T)}-K\right) f(\mathbf{X}) \mathrm{d} \mathbf{X},
$$


where

$$
\begin{aligned}
h \equiv & \sum_{l=1}^{M} b_{l} X_{l}(T)-\sum_{l=M+1}^{M+N} b_{l} X_{l}(T)+\tilde{F} \\
& -\ln \phi_{T}\left(u_{1}=-i b_{1}, \cdots, u_{M}=-i b_{M}, u_{M+1}=0, \cdots, u_{M+N}=0\right) \\
& +\ln \phi_{T}\left(u_{1}=0, \cdots, u_{M}=0, u_{M+1}=-i b_{M+1}, \cdots, u_{M+N}=-i b_{M+N}\right) .
\end{aligned}
$$

Now, the inner integral in $\tilde{K}$ can be computed explicitly and it reads as

$$
\begin{aligned}
& \frac{1}{i \gamma+\alpha} \exp \left[( i \gamma + \alpha ) \left(\sum_{l=1}^{M} b_{l} X_{l}(T)-\sum_{l=M+1}^{M+N} b_{l} X_{l}(T)+\tilde{F}\right.\right. \\
& -\ln \phi_{T}\left(u_{1}=-i b_{1}, \cdots, u_{M}=-i b_{M}, u_{M+1}=0, \cdots, u_{M+N}=0\right) \\
& \left.\left.+\ln \phi_{T}\left(u_{1}=0, \cdots, u_{M}=0, u_{M+1}=-i b_{M+1}, \cdots, u_{M+N}=-i b_{M+N}\right)\right)\right] .
\end{aligned}
$$

Therefore, $\Psi_{T}(\gamma ; \alpha, \tilde{F}, \tilde{K}, \mathbf{b})$ can be re-written as:

$$
\begin{aligned}
& \int_{\mathbb{R}^{M+N}} \frac{1}{i \gamma+\alpha} \times \exp \left[( i \gamma + \alpha ) \left(\sum_{l=1}^{M} b_{l} X_{l}(T)-\sum_{l=M+1}^{M+N} b_{l} X_{l}(T)+\tilde{F}\right.\right. \\
& -\ln \phi_{T}\left(u_{1}=-i b_{1}, \cdots, u_{M}=-i b_{M}, u_{M+1}=0, \cdots, u_{M+N}=0\right) \\
& \left.\left.+\ln \phi_{T}\left(u_{1}=0, \cdots, u_{M}=0, u_{M+1}=-i b_{M+1}, \cdots, u_{M+N}=-i b_{M+N}\right)\right)\right] \\
& \times\left(\sum_{l=1}^{M} w_{l} \mathrm{e}^{X_{l}(T)}-\sum_{l=M+1}^{M+N} w_{l} \mathrm{e}^{X_{l}(T)}-K\right) \times f(\mathbf{X}) \mathrm{d} \mathbf{X},
\end{aligned}
$$

i.e.

$$
\begin{aligned}
\Psi_{T}(\gamma ; \alpha, \tilde{F}, \tilde{K}, \mathbf{b})= & \frac{\mathrm{e}^{(i \gamma+\alpha)\left(\ln \frac{\Phi_{T}\left(0, \cdots, 0,-i b_{M+1} \cdots,-i b_{M+N}\right)}{\Phi_{T}\left(-i i_{1}, \cdots,-i b_{M}, 0, \cdots, 0\right)}+\tilde{F}\right)}}{i \gamma+\alpha} \\
& \times \int_{\mathbb{R}^{M+N}}\left[\exp \left((i \gamma+\alpha) \times\left(\sum_{l=1}^{M} b_{l} X_{l}(T)-\sum_{l=M+1}^{M+N} b_{l} X_{l}(T)\right)\right)\right. \\
& \left.\times\left(\sum_{l=1}^{M} w_{l} \mathrm{e}^{X_{l}(T)}-\sum_{l=M+1}^{M+N} w_{l} \mathrm{e}^{X_{l}(T)}-K\right) f(\mathbf{X})\right] \mathrm{d} \mathbf{X} .
\end{aligned}
$$

Finally, from the definition of the characteristic function for the vector $\mathbf{X}(T)$, see Equation (5), we have that

$$
\begin{aligned}
& \Psi_{T}(\gamma ; \alpha, \tilde{F}, \tilde{K}, \mathbf{b})=\frac{\exp \left(i(\gamma-\alpha i)\left(\tilde{F}+\ln \left(\frac{\phi_{T}\left(0, \cdots, 0,-i b_{M+1}, \cdots,-i b_{M+N}\right)}{\phi_{T}\left(-i b_{1}, \cdots,-i b_{M}, 0, \cdots, 0\right)}\right)\right)\right)}{i(\gamma-\alpha i)} \\
& \times\left(\sum_{j=1}^{M} w_{j} \phi_{T}\left((\gamma-\alpha i) b_{1}-i \delta_{1 j}, \cdots,(\gamma-\alpha i) b_{M}-i \delta_{M j},-(\gamma-\alpha i) b_{M+1}, \cdots,-(\gamma-\alpha i) b_{M+N}\right)\right. \\
& -\sum_{j=M+1}^{M+N} w_{j} \phi_{T}\left((\gamma-\alpha i) b_{1}, \cdots,(\gamma-\alpha i) b_{M},-(\gamma-\alpha i) b_{M+1}-i \delta_{(M+1) j}, \cdots,-(\gamma-\alpha i) b_{M+N}-i \delta_{(M+N) j}\right) \\
& \left.-K \phi_{T}\left((\gamma-\alpha i) b_{1}, \cdots,(\gamma-\alpha i) b_{M},-(\gamma-\alpha i) b_{M+1}, \cdots,-(\gamma-\alpha i) b_{M+N}\right)\right),
\end{aligned}
$$

where $\delta_{i j}$ is the Kronecker delta and where $\tilde{F}, \tilde{K}$ and the coefficients 
$\mathbf{b}=\left(b_{1}, \cdots, b_{M+N}\right)^{\mathrm{T}}$ are defined in Equations (3), (4) respectively.

This concludes the proof.

\section{A.2. Proof of Proposition 3}

Proof. In the multi-variate Black and Scholes model, see [1], an explicit solution of Equation (22) is given by:

$$
S_{k}(T)=S_{k}(t) \exp \left(\left(r-q_{k}-\frac{\sigma_{k}^{2}}{2}\right)+\sigma_{k}\left(W_{k}(T)-W_{j}(t)\right)\right), j=1, \cdots, M+N .
$$

We denote the joint distribution of the multi-variate random vector

$$
\mathbf{S}(T)=\left(S_{1}(T), \cdots, S_{N+M}(T)\right)^{\mathrm{T}}
$$

as $\operatorname{MLN}(\mathbf{m}, V)$, namely as a multi-variate lognormal distribution with mean vector $\mathbf{m}$ and covariance matrix $V$, where:

$$
\mathbf{m}=\left(m_{k}\right)_{k=1, \cdots, N+M}=\left(\ln S_{k}(t)+\left(r-q_{k}-\frac{\sigma_{k}^{2}}{2}\right)(T-t)\right)_{k=1, \cdots, M+N}
$$

and

$$
V=(T-t)\left(v_{k l}\right)_{k, l=1, \cdots, M+N}, \text { with } v_{k l}= \begin{cases}\sigma_{k}^{2}, & \text { for } k=l, \\ \rho_{k l} \sigma_{k} \sigma_{l} n, & \text { otherwise. }\end{cases}
$$

Define

$$
R(T) \equiv \frac{\prod_{k=1}^{M} S_{k}(T)^{b_{k}}}{\prod_{k=M+1}^{M+N} S_{k}(T)^{b_{k}}} .
$$

Straightforward calculations show that

$$
\begin{aligned}
R(T)= & \frac{\prod_{k=1}^{M} S_{k}(T)^{b_{k}}}{\prod_{k=M+1}^{M+N} S_{k}(T)^{b_{k}}} \times \exp \left((T-t)\left[\sum_{k=1}^{M} b_{k}\left(r-q_{k}-\frac{\sigma_{k}^{2}}{2}\right)-\sum_{k=M+1}^{M+N} b_{k}\left(r-q_{k}-\frac{\sigma_{k}^{2}}{2}\right)\right]\right. \\
& \left.+\sum_{k=1}^{M} b_{k} \sigma_{k} W_{k}(t)-\sum_{k=M+1}^{M+N} b_{k} \sigma_{k} W_{k}(t)\right) .
\end{aligned}
$$

Then, we set

$$
Z \equiv \frac{\sum_{k=1}^{M} b_{k} \sigma_{k} W_{k}(t)-\sum_{k=M+1}^{M+N} b_{k} \sigma_{k} W_{k}(t)}{\sqrt{T-t} \sigma_{R}},
$$

with $Z \sim N(0,1)$, and where

$$
\sigma_{R}^{2}=\sum_{i=1}^{M+N} \sum_{j=1}^{M+N} \rho_{i j} \sigma_{i} \sigma_{j} m_{i} m_{j}, \quad \text { with } m_{i}=\left\{\begin{array}{l}
\frac{F_{i}(t, T)}{\mathrm{e}^{\tilde{F}}}, i=1, \cdots, M, \\
-\frac{F_{i}(t, T)}{\mathrm{e}^{\tilde{K}}}, i=M+1, \cdots, M+N,
\end{array}\right.
$$


where

$$
\tilde{F}=\ln \left(\sum_{i=1}^{M} F_{i}(t, T)\right) \text {, and } \tilde{K}=\ln \left(\sum_{i=M+1}^{M+N} F_{i}(t, T)+K\right) \text {. }
$$

Therefore, we can re-write the set $A$ as follows:

$$
A=\left\{\omega: R(T)>\frac{\mathrm{e}^{\tilde{K}-\tilde{F}} \mathbb{E}^{\mathbb{Q}}\left[\prod_{k=1}^{M} S_{k}(T)^{b_{k}}\right]}{\mathbb{E}^{\mathbb{Q}}\left[\prod_{k=M+1}^{M+N} S_{k}(T)^{b_{k}}\right]}\right\}=\{\omega: Z \geq d\},
$$

where

$$
\begin{aligned}
d= & \frac{1}{\sqrt{T-t} \sigma_{R}}\left(\tilde{K}-\tilde{F}-\ln \left(R(t) \mathbb{E}^{\mathbb{Q}}\left[\prod_{k=M+1}^{M+N} S_{k}(T)^{b_{k}}\right]\right)+\ln \left(\mathbb{E}^{\mathbb{Q}}\left[\prod_{k=1}^{M} S_{k}(T)^{b_{k}}\right]\right)\right. \\
& \left.-(T-t)\left(\sum_{k=1}^{M} b_{k}\left(r-q_{k}-\frac{\sigma_{k}^{2}}{2}\right)-\sum_{k=M+1}^{M+N} b_{k}\left(r-q_{k}-\frac{\sigma_{k}^{2}}{2}\right)\right)\right) .
\end{aligned}
$$

If we define $U \equiv \sum_{k=1}^{M} w_{k} S_{k}(T)-\sum_{k=M+1}^{M+N} w_{k} S_{k}(T)-K$, then the extended Bjerksund and Stensland lower bound can be equivalently re-written as

$$
\mathbb{E}\left[U^{+}\right] \geq \mathbb{E}\left[U \mathbb{1}_{\{A\}}\right]^{+}=\mathbb{E}\left[\mathbb{E}[U \mid Z] \mathbb{1}_{\{Z \geq d\}}\right]^{+}
$$

The following result will be used.

Proposition 6 (Distribution of a Multivariate Normal Distribution). Let $\boldsymbol{X} \sim N_{n}(\mu, \Sigma)$, such that $\boldsymbol{X}$ is partitioned as follows.

$$
\boldsymbol{X}=\left(\begin{array}{l}
X_{1} \\
X_{2}
\end{array}\right) \text { with sizes }\left(\begin{array}{c}
q \times 1 \\
(n-q) \times 1
\end{array}\right)
$$

and accordingly $\mu$ and $\Sigma$ are partitioned as follows:

$$
\begin{gathered}
\mu=\left(\begin{array}{l}
\mu_{1} \\
\mu_{2}
\end{array}\right) \text {, with sizes }\left(\begin{array}{c}
q \times 1 \\
(n-q) \times 1
\end{array}\right), \\
\Sigma=\left(\begin{array}{cc}
\Sigma_{11} & \Sigma_{12} \\
\Sigma_{21} & \Sigma_{22}
\end{array}\right) \text { with sizes }\left(\begin{array}{cc}
q \times q & q \times(n-q) \\
(n-q) \times q & (n-q) \times(n-q)
\end{array}\right),
\end{gathered}
$$

where $\left|\Sigma_{22}\right|>0$.

Then, the distribution of $X_{1}$, conditional on $X_{2}=x_{2}$, is a multivariate normal, namely

$$
X_{1} \mid X_{2}=x_{2} \sim N_{q}(\tilde{\mu}, \tilde{\Sigma})
$$

where

$$
\tilde{\mu}=\mu_{1}+\Sigma_{12} \Sigma_{22}^{-1}\left(x_{2}-\mu_{2}\right),
$$

and

$$
\tilde{\Sigma}=\Sigma_{11}-\Sigma_{12} \Sigma_{22}^{-1} \Sigma_{21} .
$$


Note that the covariance does not depend on the value $x_{2}$ of the conditioning variable.

Proof. See [40].

If we look at the definition of the random variable $Z$ in Equation (33), this can be re-written in a more convenient way as follows:

$$
Z=\mathbf{a}^{\mathrm{T}} \cdot \mathbf{W}(T-t)
$$

where

$$
\mathbf{a}^{\mathrm{T}} \equiv\left(\frac{b_{1} \sigma_{1}}{\sigma_{R} \sqrt{T-t}}, \cdots, \frac{b_{M} \sigma_{M}}{\sigma_{R} \sqrt{T-t}}, \frac{-b_{M+1} \sigma_{M+1}}{\sigma_{R} \sqrt{T-t}}, \cdots, \frac{-b_{M+N} \sigma_{M+N}}{\sigma_{R} \sqrt{T-t}}\right)^{\mathrm{T}},
$$

and from the property related to affine transformations of multivariate normal distribution, we know that, if $\mathbf{X} \sim N_{n}\left(\mu_{\mathbf{X}}, \Sigma_{\mathbf{X}}\right)$, then

$$
\mathbf{a}^{\mathrm{T}} \cdot \mathbf{X} \sim N_{1}\left(\mathbf{a}^{\mathrm{T}} \cdot \mu_{\mathrm{X}}, \mathbf{a}^{\mathrm{T}} \Sigma_{\mathbf{X}} \mathbf{a}^{\mathrm{T}}\right), \forall \mathbf{a} \in \mathbb{R}^{n} .
$$

This holds in particular for $\mathbf{W}(T-t)$, since

$$
\mathbf{W}(T-t) \sim N_{N+M}\left(\mu_{\mathbf{W}}=\mathbf{0}, \Sigma_{\mathbf{W}}=(T-t)\left(\rho_{i j}\right)_{i, j=1, \cdots, N+M}\right) .
$$

Besides that, we have

$$
\begin{aligned}
& \operatorname{Cov}\left(\mathbf{a}^{\mathrm{T}} \cdot \mathbf{W}(T-t), W_{k}(T-t)\right) \\
& = \begin{cases}\frac{\sqrt{T-t}}{\sigma_{R}}\left(\sigma_{k} b_{k}+\sum_{l=1(l \neq k)}^{M} \rho_{k l} \sigma_{l} b_{l}-\sum_{l=M+1}^{M+N} \rho_{k l} \sigma_{l} b_{l}\right), & \text { for } k=1, \cdots, M, \\
\frac{\sqrt{T-t}}{\sigma_{R}}\left(-\sigma_{k} b_{k}+\sum_{l=1}^{M} \rho_{k l} \sigma_{l} b_{l}-\sum_{l=M+1(l \neq k)}^{M+N} \rho_{k l} \sigma_{l} b_{l}\right), & \text { for } k=M+1, \cdots, M+N .\end{cases}
\end{aligned}
$$

Therefore, the vector $\left(W_{1}(T-t), \cdots, W_{N+M}(T-t), \mathbf{a}^{\mathrm{T}} \cdot \mathbf{W}(T-t)\right)^{\mathrm{T}}$ is normally distributed with null mean vector $\mu_{\mathbf{W}, \mathbf{a}^{\mathrm{T}} \cdot \mathbf{W}}=\mathbf{0}$ and covariance matrix $\Sigma_{\mathbf{W}, \mathbf{a}^{\mathrm{T}} \cdot \mathbf{W}}$, which can be written in the following form:

$$
\Sigma_{\mathbf{W}, \mathbf{a}^{\mathrm{T}} \cdot \mathbf{W}}=\left(\begin{array}{cc}
\Sigma_{11} & \Sigma_{12} \\
\Sigma_{21} & \Sigma_{22}
\end{array}\right)
$$

where $\Sigma_{11} \equiv \Sigma_{\mathbf{w}}, \quad \Sigma_{22} \equiv 1$ and where

$$
\Sigma_{12}=\left(\Sigma_{12}\right)_{k, l=1, \cdots, N+M}=\left(\operatorname{Cov}\left(\mathbf{a}^{\mathrm{T}} \cdot \mathbf{W}(T-t), W_{k}(T-t)\right)\right)_{k, l=1, \cdots, N+M}
$$

and $\Sigma_{21}=\Sigma_{12}^{\mathrm{T}}$.

By applying the results in Proposition 6, we conclude that the distribution of the vector $\left(W_{1}(T-t), \cdots, W_{N+M}(T-t)\right)^{\mathrm{T}}$, conditional on $\mathbf{a}^{\mathrm{T}} \cdot \mathbf{W}(T-t)$, is normal with mean vector $\tilde{\mu}$ and covariance matrix $\tilde{\Sigma}$, respectively given by:

$$
\tilde{\mu}=\sqrt{T-t} Z \mathbf{c} \text {, and } \tilde{\Sigma}=\Sigma_{\mathbf{w}}-(T-t) \mathbf{c c}^{\mathrm{T}} \text {. }
$$

where 


$$
c_{k}= \begin{cases}\frac{1}{\sigma_{R}}\left(\sigma_{k} b_{k}+\sum_{l=1(l \neq k)}^{M} \rho_{k l} \sigma_{l} b_{l}-\sum_{l=M+1}^{M+N} \rho_{k l} \sigma_{l} b_{l}\right), & \text { for } k=1, \cdots, M, \\ \frac{1}{\sigma_{R}}\left(-\sigma_{k} b_{k}+\sum_{l=1}^{M} \rho_{k l} \sigma_{l} b_{l}-\sum_{l=M+1(l \neq k)}^{M+N} \rho_{k l} \sigma_{l} b_{l}\right), & \text { for } k=M+1, \cdots, M+N .\end{cases}
$$

and therefore, it follows that $\left(S_{1}(T), \cdots, S_{N+M}(T) \mid Z\right)^{T} \sim M L N(\tilde{m}, \tilde{V})$, where

$$
\tilde{m}=\left(\ln S_{k}(t)+\left(r-q_{k}-\frac{1}{2} \sigma_{k}^{2}\right)(T-t)\right)_{k=1, \cdots, M+N}
$$

and

$$
\left\{\begin{array}{l}
\tilde{V}_{k k}=\left(\sigma_{k}^{2}\left(1-c_{k k}^{2}\right)\right), \quad \text { for } k=1, \cdots, N+M \\
\tilde{V}_{k l}=\left(\sigma_{k} \sigma_{l}\left(\rho_{k l}-c_{k} c_{l}\right)\right), \quad \text { for } k \neq l=1, \cdots, N+M .
\end{array}\right.
$$

We can now compute the approximated payoff expectation in Equation (34) as follows:

$$
\begin{aligned}
\mathbb{E} & {\left[\mathbb{E}[U \mid Z] \mathbb{1}_{\{Z \geq d\}}\right]^{+} } \\
= & \mathbb{E}^{\mathbb{Q}}\left[\left(\sum_{k=1}^{M} w_{k} \exp \left\{\ln \left(S_{k}(t)\right)+\left(r-q_{k}-\frac{1}{2} \sigma_{k}^{2} c_{k}^{2}\right)(T-t)+\sigma_{k} c_{k} \sqrt{T-t} Z\right\}\right.\right. \\
& \left.\left.\left.-\sum_{k=M+1}^{M+N} w_{k} \exp \left\{\ln \left(S_{k}(t)\right)+\left(r-q_{k}-\frac{1}{2} \sigma_{k}^{2} c_{k}^{2}\right)(T-t)+\sigma_{k} c_{k} \sqrt{T-t} Z\right\}-K\right\}\right) \nVdash_{\{Z \geq d\}}\right]^{+}
\end{aligned}
$$

By using the partial expectation property of the lognormal distribution ${ }^{3}$ and discounting, the above expectation gives us the Extended Bjerksund and Stensland pricing formula:

$$
\begin{aligned}
C(t, T, K)^{E B S}= & \mathrm{e}^{-r(T-t)}\left(\sum_{k=1}^{M} w_{k} S_{k}(t) \mathrm{e}^{\left(r-q_{k}\right)(T-t)} N\left(\sigma_{k} c_{k} \sqrt{T-t}-d\right)\right. \\
& \left.-\sum_{k=M+1}^{M+N} w_{k} S_{k}(t) \mathrm{e}^{\left(r-q_{k}\right)(T-t)} N\left(\sigma_{k} c_{k} \sqrt{T-t}-d\right)-K N(-d)\right)
\end{aligned}
$$

where $N(\cdot)$ is the cumulative distribution function for a standard normal variable.

The coefficients $b_{1}, \cdots, b_{N+M}$ as well as $\tilde{F}$ and $\tilde{K}$, appearing in the definition of c and $d$, are set by generalizing the guess of Bjerksund and Stensland in [30] for the two-asset spread options pricing problem.

This concludes the proof.

${ }^{3}$ This property states that for a log-normal random variable $X$ with parameters $\mu$ and $\sigma$, we have that $\mathbb{E}[X \mid X>s]=\mathrm{e}^{\mu+\frac{1}{2} \sigma^{2}} N\left(\frac{\mu+\sigma^{2}-\ln s}{\sigma}\right)$. 
Submit or recommend next manuscript to SCIRP and we will provide best service for you:

Accepting pre-submission inquiries through Email, Facebook, LinkedIn, Twitter, etc. A wide selection of journals (inclusive of 9 subjects, more than 200 journals)

Providing 24-hour high-quality service

User-friendly online submission system

Fair and swift peer-review system

Efficient typesetting and proofreading procedure

Display of the result of downloads and visits, as well as the number of cited articles

Maximum dissemination of your research work

Submit your manuscript at: http://papersubmission.scirp.org/

Or contact jmf@scirp.org 